\title{
Evidence-Based Updates to Thrombectomy: Targets, New Techniques, and Devices
}

\author{
Leonard L. L. Yeo 1,2*, Mingxue Jing 1,2, Pervinder Bhogal ${ }^{3}$, Tianming Tu ${ }^{4}$, \\ Anil Gopinathan ${ }^{1,5}$, Cunli Yang ${ }^{1,5}$, Benjamin Y. Q. Tan ${ }^{1,2}$, Fabian Arnberg ${ }^{6}$, Ching-Hui Sia ${ }^{1,7}$, \\ Staffan Holmin ${ }^{6}$ and Tommy Andersson ${ }^{6,8}$ \\ ${ }^{1}$ Yong Loo Lin School of Medicine, National University of Singapore, Singapore, Singapore, ${ }^{2}$ Division of Neurology, \\ Department of Medicine, National University Health System, Singapore, Singapore, ${ }^{3}$ Department of Neuroradiology, St. \\ Bartholomew's and the Royal London Hospital, London, United Kingdom, ${ }^{4}$ Department of Neurology, National Neuroscience \\ Institute, Singapore, Singapore, ${ }^{5}$ Department of Diagnostic Imaging, National University Health System, Singapore, \\ Singapore, ${ }^{6}$ Department of Clinical Neuroscience, Karolinska Institutet and Department of Neuroradiology, Karolinska \\ University Hospital, Stockholm, Sweden, ${ }^{7}$ Department of Cardiology, National University Heart Centre, Singapore, \\ Singapore, ${ }^{8}$ Department of Medical Imaging, AZ Groeninge, Kortrijk, Belgium
}

OPEN ACCESS

Edited by:

Johanna Ospel,

University Hospital of

Basel, Switzerland

Reviewed by:

Petra Cimflova

University of Calgary, Canada

Nabeel A. Herial,

Thomas Jefferson University,

United States

Adrien Guenego,

Stanford Healthcare, United States

*Correspondence:

Leonard L. L. Yeo

leonardyeol/@gmail.com

Specialty section:

This article was submitted to Endovascular and Interventional Neurology, a section of the journa Frontiers in Neurology

Received: 20 May 2021

Accepted: 30 July 2021

Published: 09 September 2021

Citation:

Yeo LLL, Jing M, Bhogal P, Tu T, Gopinathan A, Yang C, Tan BYQ,

Arnberg F, Sia C-H, Holmin S and Andersson T (2021) Evidence-Based Updates to Thrombectomy: Targets,

New Techniques, and Devices.

Front. Neurol. 12:712527.

doi: 10.3389/fneur.2021.712527
Endovascular thrombectomy (EVT) has been validated in several randomized controlled trials in recent years for its efficacy in the treatment of acute ischemic strokes (AIS) and is now the standard of care according to international guidelines. However, in about $20 \%$ of EVT procedures, recanalization is not achieved, and over $50 \%$ of patients who undergo EVT still do not have good functional outcome. In this article, we provide an extensive review of the latest evidence and developments in the field of EVT, with particular focus on the factors that improve patient outcomes. These factors include new and adjunctive techniques such as combination of direct aspiration and stent retriever, intra-arterial urokinase or 2b/3a inhibitors, rescue stenting, as well as novel devices including balloon guide catheters and the newer generations of aspiration catheters and stent retrievers. We also examined the latest notion of using first-pass effect (FPE) as the target to achieve during EVT, which has been associated with an improved functional outcome. While the field of EVT has been rapidly evolving, further research is required in specific AIS patient populations such as those with large ischemic core, late presentation beyond $24 \mathrm{~h}$, posterior circulation strokes, and with distal medium vessel occlusion or tandem lesions to better assess its efficacy and safety.

Keywords: acute stroke, ischaemic, thrombectomy, stent retriever, aspiration, devices, reperfusion

\section{INTRODUCTION}

In recent years, several randomized clinical trials in acute ischemic stroke (AIS) have validated and cemented the efficacy of endovascular thrombectomy (EVT) in proximal anterior circulation occlusions (1-6). This revolutionary treatment modality has now emerged as the standard of care in international guidelines and is considered level 1 class A evidence $(7,8)$. Although EVT has strong evidence for the treatment of AIS, it is unable to achieve recanalization in approximately $20 \%$ of AIS patients $(9,10)$. Moreover, up to half of the patients who undergo EVT still do not have a good functional outcome at 3 months, and this includes patients who do have good reperfusion (6). Nonetheless, the field continues to evolve rapidly, with constant innovations. In this review, we 
attempt to summarize the latest evidence-based developments to the field of EVT in the context of AIS, in an attempt to determine what will allow us to improve patient outcomes.

\section{TARGETS}

\section{eTICl as Measurement of Success in Thrombectomy}

The initial measure of EVT success was extrapolated from cardiology radiological results with the thrombolysis in myocardial infarction score (TIMI). This was quickly adapted into a more cerebral circulation-based thrombolysis in cerebral infarction (TICI) score and subsequently the modified thrombolysis in cerebral infarction score (mTICI) where a mTICI $2 b$ or greater score, equivalent to $>50 \%$ reperfusion of the affected territory, is considered a successful reperfusion for EVT (11).

Recently, a group of authors examined the data from the HERMES trials and proposed a revised TICI grading scale, the expanded TICI (eTICI). This is a 7-point grade from eTICI 0 to eTICI 3 which assessed the degree of reperfusion in a more quantitative manner by splitting the TICI $2 \mathrm{~b}$ into more granular divisions (12). In brief, eTICI grade 0 is equivalent to no reperfusion or $0 \%$ filling of the downstream territory; eTICI 1 reflects thrombus reduction without any reperfusion of distal arteries; eTICI $2 \mathrm{a}$ is reperfusion of less than half or $1-49 \%$ of the territory; eTICI $2 \mathrm{~b} 50$ is $50-66 \%$ reperfusion (Figure 1A), eTICI 2 b67 is $67-89 \%$ reperfusion, exceeding TICI $2 \mathrm{~B}$ but below TICI 2C (Figure 1B), eTICI $2 \mathrm{c}$ is equivalent to TICI $2 \mathrm{C}$ which represents $90-99 \%$ reperfusion or near complete; and eTICI 3 is complete or $100 \%$ reperfusion, similar to TICI 3. The authors found that after adjustment of covariates, eTICI remained an independent predictor of outcome on multivariate analysis of the mRS shift, and more importantly, adjacent categories of 2a, 2b50, and 2 b67 are important distinctions with clinical implications.

In previous established consensus recommendations for reperfusion target in endovascular thrombectomy, successful reperfusion was defined as exceeding $50 \%$ of the territory (13). Now with evidence that within the $>50 \%$ reperfusion category, further subdivisions into $50-66 \%, 67-89 \%$, and $90-99 \%$ help to identify meaningful differences in clinical outcomes, the authors of the paper therefore proposed to adopt eTICI 2 b67 as the ideal threshold for defining successful reperfusion. Moving forward, considerations should be made to adopt a more granular scale which has better prognostic value and clinical utility.

\section{First-Pass Recanalization: A New Target for Thrombectomy}

With the advent of better equipment and improved devices for EVT, there have been increasingly better rates of successful reperfusion. Accordingly, the standard of care is constantly being re-evaluated to determine what is an achievable benchmark for AIS LVO patients. Recently, there has been a move to nominate a single pass-reperfusion standard as the target to achieve during EVT. Several studies and a recent meta-analysis have shown that there is clinical rationale behind this first-pass reperfusion with improved functional outcomes and such successful initial attempts have been termed first-pass effect (FPE) (14). In the aforementioned meta-analysis which composed 21 studies and 2,747 patients, FPE patients not only displayed better functional outcomes but also had lower mortality rates than patients who did not have FPE. Interestingly, they also showed that complete reperfusion with a single pass (FPE-mTICI 3) was associated with better 3-month outcomes compared with FPE-mTICI 2B (mRS $0-2$, 66 vs. $46 \%$; OR, 0.46; 95\% CI, 0.037-0.57), better mortality rates ( 8 vs. $14 \%$ ) and less intracranial hemorrhage (22 vs. $31 \%$ ). However, it should be said that while FPE is desirable and clinical outcomes worsen with more attempts, the goal of thrombectomy is to recanalize the vessel, and recanalization after multiple passes is better than no recanalization at all (15).

Besides being used clinically, FPE mTICI $\geq 2 \mathrm{~B}$ is increasingly being used to evaluate thrombectomy devices as a new benchmark. This is because in the latest trials, devices achieve $>90 \%$ reperfusion rates, and there is very little differentiating them (16-18). A new threshold, FPE mTICI $\geq 2 \mathrm{~B}$ or even FPE mTICI 2c-3, with its marked clinical improvement, is a potential way to evaluate these devices and tease apart their small differences, as we strive to achieve perfect stroke outcomes.

\section{TECHNIQUES}

\section{Direct Aspiration vs. Stent Retriever: ASTER and COMPASS Trial}

The two most common EVT techniques for reperfusion are via a stent retriever or aspiration with a large bore catheter. It remains a matter of debate as to which technique is superior, The Contact Aspiration vs. Stent Retriever for Successful Revascularization (ASTER) study was a randomized, open-label, blinded endpoint superiority trial designed to address this problem. In this study, 381 patients were recruited, with 192 patients assigned first-line direct aspiration and 189 assigned to first-line stent retriever use. Successful reperfusion was achieved at similar rates with direct aspiration (85.4\%) and stent retrievers (83.1\%), $p=0.53$. Nonetheless, this was a failed superiority trial that was underpowered to demonstrate a significant difference between the two techniques (17).

After the failure of the ASTER trial, the similar reperfusion rates between modalities led a North American group to change track and conduct a non-inferiority trial in 15 North American sites to once again compare the efficacy between large bore direct aspiration and stent retrievers. In this trial entitled COMPASS, 270 patients without a large early infarct core (ASPECTS $>6$ ) and who presented within $6 \mathrm{~h}$ of onset were enrolled. Ultimately, 134 received direct aspiration as first-line treatment and 136 received stent retriever use as first-line treatment. Direct aspiration achieved $52 \%$ good functional outcomes at 3 months which was comparable with the $50 \%$ achieved by first-line stent retriever use and reached non-inferiority in the analysis $(p=0.0014)(18)$. This trial was the landmark trial to provide level 1 evidence in support of direct aspiration, and the study authors also discussed that even in the event of failure of direct aspiration, the large 

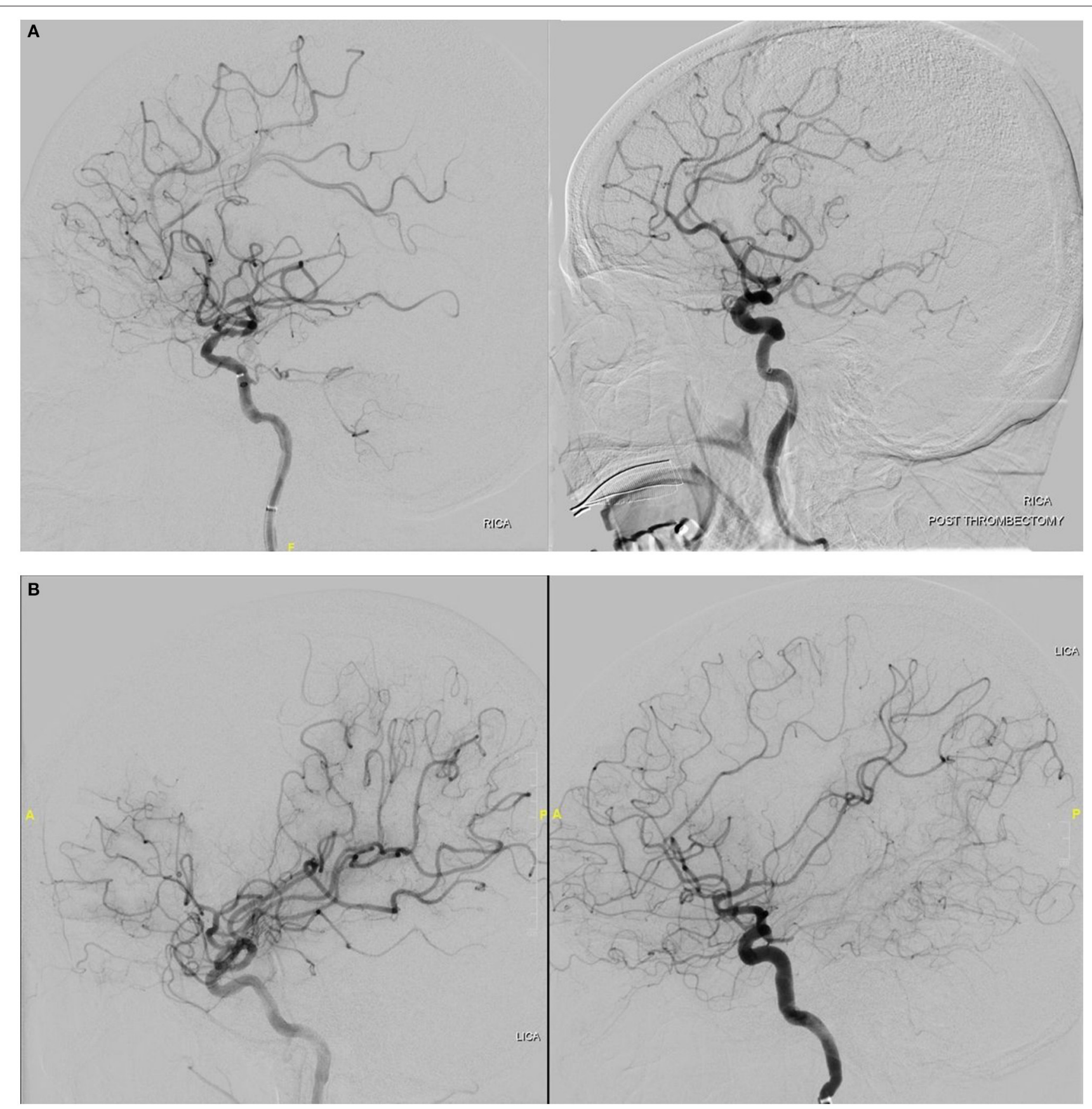

FIGURE 1 | (A) Cerebral digital subtraction angiography showing eTICl 2b50 recanalization in a patient with right MCA occlusion. (B) Cerebral digital subtraction angiography showing eTICl $2 \mathrm{~b} 67$ recanalization in a patient with left MCA occlusion.

bore catheter is still at the clot face and a stent retriever can be quickly deployed over the thrombus. This resulted in the initial direct aspiration arm showing a significantly shorter procedural time than stent retriever use. There was also a mention of the additional benefit that aspiration catheters tend to be more affordable than stent retrievers. Nonetheless, the authors stressed that EVT technique should be tailored to the individual patient and clot characteristics for maximal efficacy.

\section{Combined Techniques}

Thrombectomy in many centers are now performed with the combination of a stent retriever and a distal aspiration catheter as well as a balloon guide catheter. These range from the stent retriever-assisted vacuum-locked extraction (SAVE) technique, the BAlloon guiDe with large bore Distal Access catheter with dual aspiration with Stent Retriever as Standard (BADDASS) approach, the aspiration-retriever technique for stroke (ARTS), a 
stent retrieving into an aspiration catheter with proximal balloon technique (ASAP), or proximal balloon occlusion together with direct thrombus aspiration during stent retriever thrombectomy (PROTECT-PLUS) (19-24). These are several different variations on the same techniques for thrombectomy, all of which report a high rate of reperfusion, higher first-pass recanalization rates, lower number of attempts, and/or a lower rate of distal embolization. While it may be more expensive to use more equipment, this is offset by the improved first-pass recanalization rate and shorter procedure times which lead to better outcomes.

\section{Adjunctive Intra-Arterial Treatment: Urokinase, tPA, and $2 b / 3 a$ Inhibitors}

The Prolyse in Acute Cerebral Thromboembolism (PROACT) II trial is an early study conducted before the use of stent retrievers or aspiration catheters. It provided some grounds for intra-arterial infusion of recombinant pro-urokinase resulting in recanalization in approximately $2 / 3$ of stroke patients with MCA occlusions (25). However, there was a five times greater risk of symptomatic intracranial hemorrhage ( $\mathrm{SICH})$ with prourokinase and production was stopped before it could obtain US FDA approval as a thrombolytic agent. It has since gradually fallen out of use in the USA, although it is still commonly used in other parts of the world.

The use of thrombolytic agents in conjunction with other thrombectomy methods has rarely been described. If urokinase has the ability to treat microthrombi, it may be able to improve forward flow and perfusion which in turn could contribute to a reduction in infarct size and rate of SICH (26). In one study where urokinase was used in 15 patients in conjunction with thrombectomy, reperfusion was improved in eight patients (53.3\%) (27). In other studies, the risk of bleeding and SICH did not appear to be increased with adjunctive tPA or urokinase (28-31). A larger study including 100 patients out of a total cohort of 991 patients who received intra-arterial urokinase administered during mechanical thrombectomy was recently published. While patients who had unsuccessful thrombectomy were included, the most common reason for instillation of intraarterial (IA) urokinase in this study was incomplete reperfusion, which was defined as mTICI $<3$ and seen in 53 (53\%) patients. The 100 patients treated with IA urokinase during EVT did not demonstrate an increased risk of SICH or mortality. Interestingly in the 53 patients with incomplete reperfusion, there was a significantly higher rate of mRS 0-2 at 3 months (27). This study provides the groundwork for the potential use of urokinase in incomplete reperfusion and may help in improving the chance for reperfusion in distal eloquent occlusions or tortuous distal anatomy. Ultimately, it will need to be validated in larger studies such as the upcoming Multi-arm Optimization of Stroke Thrombolysis (MOST) study (32).

\section{DEVICES}

\section{Balloon Guide Catheter}

The balloon guide catheter (BGC) is a simple upgrade from the typical guide catheter with a large lumen $(6-9 F)$ and an inflatable balloon on the distal tip of the catheter. It is used to generate flow arrest of blood and even flow reversal when negative pressure is applied to remove emboli generated during the EVT procedure and prevent embolic complications. This has been shown in vitro where experiments with a vascular phantom occlusion model showed that use of a BGC resulted in 50\% reduction of soft clot fragments compared with a conventional guide catheter (33). Similarly, in an animal porcine model, a BGC provided reproducible flow arrest with the balloon inflated, and this translated into reliable flow reversal with manual aspiration using a regular syringe. In comparison, manual aspiration with a syringe in a similar sized conventional guide catheter resulted in oscillatory flow or even an occluding collapse of the walls of the distal vessel (34).

The benefits of BGC thrombectomy has been shown in multiple different studies. In the investigator-initiated TRACK registry which audits the Trevo device, 536 anterior circulation stroke patients of whom 279 (52.1\%) had BGC placement showed that mTICI $2 \mathrm{~b}-3$ scores were higher in the BGC group (84 vs. $75.5 \%$; $p=0.01$ ) with better 3 months outcomes (57 vs. $40 \%$; $p=0.0004)$ and mortality rate (13 vs. $23 \%$; $p=0.008)$. This was despite aspiration catheter or intermediate catheter use being more common in the non-BGC group (35). In the NASA and STRATIS registries (Systematic Evaluation of Patients Treated With Neurothrombectomy Devices for Acute Ischemic Stroke), a similar effect was seen for 3-month functional outcomes. Moreover, in these two registries, the FPE was more often seen with the use of a BGC $(36,37)$. A meta-analysis of studies with BGC use which included 2,022 patients showed that BGC use was indeed associated with a higher chance of FPE (OR, 2.1; 95\% CI, 1.65-2.55) (38).

As there were certain challenges with compatibility of BCGs and large-bore distal aspiration catheters, the industry is keen on designing novel aspiration catheters that are compatible with their BCGs (e.g., novel 7Fr Catalyst fits into 8Fr Flowgate or Flowgate2 BGC). The ASTER trial documented a trend toward better mTICI 3 and better clinical outcomes in BGC-treated patients using direct aspiration (17). In the PROTECT trial with 200 patients, the group using both direct aspiration and a BGC had shorter procedure times ( 29 vs. $40 \mathrm{~min} ; p=0.002$ ), higher rate of successful recanalization (100 vs. $78 \% ; p=0.001$ ) and a higher rate of complete reperfusions (70 vs. 39\%; $p<$ 0.001) compared with sole distal aspiration during MT (22). However, larger studies are needed for confirmatory evidence to substantiate the benefits of BGC use in direct aspiration for EVT. Finally it must be mentioned that despite all purported benefits, there have been no randomized controlled trials conducted to confirm the benefits of BGC use. This is largely due to the large sample size required to show the difference and the funding required for such a trial (39).

\section{New Aspiration Catheters}

The force of aspiration is directly proportional to the inner diameter (ID) of the catheter. Prior scientific examination has similarly established the powerful relationship of ID to pressure loss and flow rate in small vessels (40-42). To take advantage of this principle, three new larger bore 0.071- to 0.072-in aspiration catheters were recently introduced for stroke thrombectomy. 

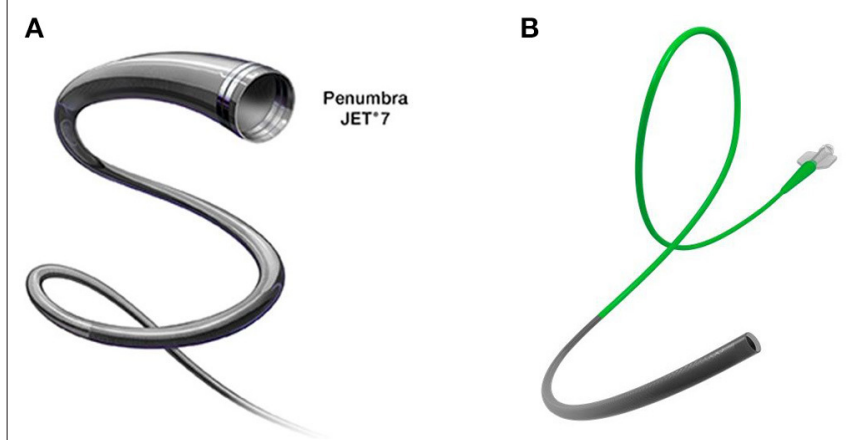

FIGURE 2 | (A) Jet 7 aspiration catheter from Penumbra with a 0.072-in $(1.83 \mathrm{~mm})$ inner lumen for greater aspiration force. (B) React 71 aspiration catheter from Medtronic (Fridley, MN, USA) with an overlapping nitinol coil and braid design for better navigability and pushability.

These catheters are named the Jet 7, the Vecta 71 , and the React 71 (Figures 2A,B). All three catheters are the largest bore direct aspiration catheters on the market that can fit within the current guide catheters and are able to generate a larger aspiration force. An early study looking at the navigability and efficacy of these aspiration catheters showed that they were able to reach the face of the clot in a high proportion (87\%) of cases: $100 \%$ with React $71,93 \%$ with Vecta 71 , and $43 \%$ with Jet $7(p=0.002)(43)$. The rate of mTICI $2 \mathrm{~b}-3$ reperfusion was also high in all three catheters and was achieved in $92 \%$ of cases: $95 \%$ with React $71,89 \%$ with Jet 7 , and $89 \%$ with Vecta 71 . These large bore catheters achieved a $39 \%$ FPE rate in this small series which provided early evidence for the use of these new catheters.

Most recently, a novel type of aspiration catheter has been introduced. The Anaconda advanced thrombectomy system (Biomed) is an aspiration catheter system that comprises a delivery catheter and a novel funnel-shaped aspiration catheter which can expand to fit the size of the vessel it is deployed in (44). The funnel shape is achieved by a stent mounted at and covered by the distal end of the catheter, which is deployed and can expand up to a maximum of $5 \mathrm{~mm}$ diameter, thus providing flow arrest for vessels of this size or smaller. This allows it to cause flow arrest akin to a BGC and consequently perform clot aspiration with a larger force as well as enable the catheter to accommodate larger clots and prevent distal emboli from fragments. It is, however, designed to be primarily used in conjunction with a stent retriever which is pulled inside the funnel that is subsequently closed and withdrawn. In an early study conducted by the company producing the device, the authors showed better first-pass reperfusion rates and fewer passes for revascularization compared with a BGC + stent retriever combination. Future independent studies will be needed to demonstrate the clinical efficacy of this new catheter device and its superiority to current aspiration thrombectomy technology.

The efficacy of aspiration catheters together with shorter procedural times and cost-effectiveness have pushed the development of even larger bore aspiration catheters. Currently, 8F 0.088-in (I.D.) aspiration catheters (Millipede 088, Perfuze

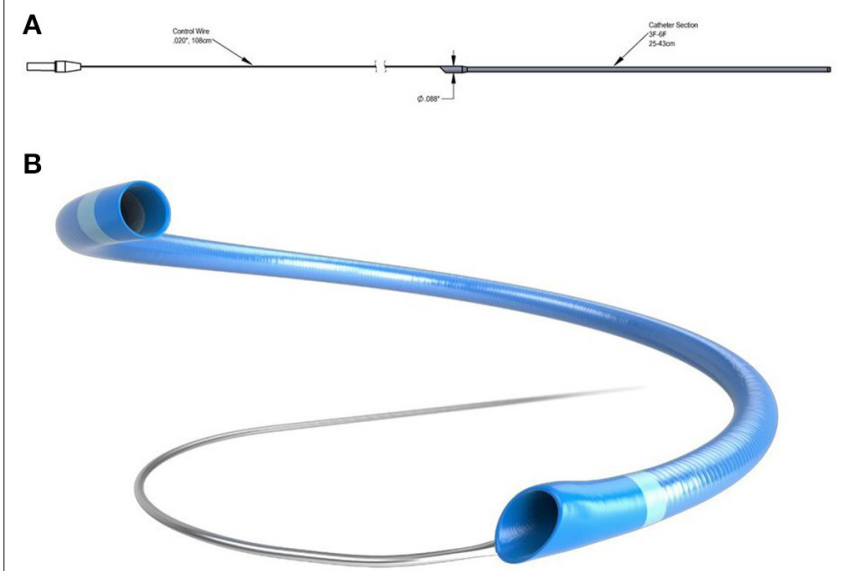

FIGURE 3 | (A,B) 6F R4Q aspiration catheter system from MIVI (Eden Prairie, $\mathrm{MN}$, USA) where the proximal portion is replaced by a wire to increase aspiration flow rates.

Ltd;) and (0.088 in, Route 92 Medical, Inc., San Mateo, CA) have been proven feasible to navigate in preclinical models of the M1 segment and the basilar artery and to be superior in clot extraction compared with smaller bore catheters (45). However, in this study, there is no published clinical data on safety and efficacy profiles of these catheters from use in patients.

Aspiration catheters typically have a tapered design where the distal tip of the aspiration catheter is slimmer than the proximal shaft, to both increase suction capability while allowing for the possibility of distal access. In the end, there is a balance between the size of the inner diameter and the wall thickness of the aspiration catheter. The $\mathrm{R}^{4} \mathrm{Q}$ aspiration catheter (MIVI Neuroscience, Inc., Eden Prairie, $\mathrm{MN}$ ) is designed to sidestep these limitations by substituting the proximal three-quarters of the catheter shaft with a stainless-steel wire (46). The $6 \mathrm{~F} \mathrm{R}^{4} \mathrm{Q}$ aspiration catheter system (Figures 3A,B) comprises a proximal pusher wire of 0.018 in and $117 \mathrm{~cm}$ length connected to a distal catheter of $25 \mathrm{~cm}$ length. It uses the guide catheter which this device is inserted into, to function as the proximal half of the catheter shaft, while the $R^{4} \mathrm{Q}$ aspiration catheter functions as the distal half of the aspiration catheter. The use of the guide catheter shaft allows it to have a larger catheter diameter with commensurately larger aspiration power. The $\mathrm{R}^{4} \mathrm{Q}$ catheters come in $6 \mathrm{~F}, 5 \mathrm{~F}, 4 \mathrm{~F}$, and $3 \mathrm{~F}$ sizes to fit to different vessel sizes. Unlike typical aspiration catheters, the suction is applied directly to the guide catheter and the $\mathrm{R}^{4} \mathrm{Q}$ can be retracted into the guide catheter. These properties have the potential to translate into clinical aspiration thrombectomy advances and the initial clinical experience with the $\mathrm{R}^{4} \mathrm{Q}$ system in a small cohort of 32 patients shows good reperfusion rates and a high rate of first-pass effect, which will need to be further validated in larger cohorts (47).

\section{Cyclical Aspiration Pumps}

Thromboaspiration for stroke thrombectomy is typically conducted by generating a static continuous vacuum either with a pump or a large syringe. In a study on cyclical aspiration, 
a SOFIA Plus catheter (MicroVention Inc., Aliso Viejo, CA) had static (29 inHg) or cyclical (18-29 inHg, $0.5 \mathrm{~Hz})$ aspiration employed using the digital CLEAR Aspiration System (Insera Therapeutics, Sacramento, CA) and eight thrombus aspiration experiments were conducted for each aspiration type in a flow model. The study showed that by varying the pressure dynamics through cyclical aspiration, it increased aspiration force on the occlusion as well as resulted in more successful clot clearance when compared with static aspiration (48). The cause of this improved ingestion may be due to the initial clot softening from dynamic compression or that dynamic friction is less than the static friction that occurs when the thrombus is stuck at the tip of the catheter. This was validated in a different study using various types of catheters with different inner diameters (0.054-0.088 in), where the use of cyclic aspiration $(18-29 \mathrm{inHg}, 0.5 \mathrm{~Hz})$ resulted in better clot ingestion into the aspiration catheter and effectively reduced the rate of distal emboli (49). More recently, in one of the first clinical experience with cyclical aspiration for largevessel strokes, investigators performed thrombectomy using the CLEAR $^{\mathrm{TM}}$ Aspiration System (Insera Therapeutics Inc., Dallas, TX) which yielded promising results. The authors reported high rate of TICI 3 FPE, which was achieved in $68.4 \%$ of the study cohort (26 of 38 patients), near-complete reperfusion (TICI $2 c / 3$ FPE) in $76.3 \%$ (29/38), and substantial reperfusion (TICI $2 b-67 / 3$ FPE) in $78.9 \%(30 / 38)$. In addition, final revascularization results for the entire cohort (in one or multiple attempts) were TICI 3 in $86.8 \%(33 / 38)$, TICI $2 c / 3$ in $94.7 \%(36 / 38)$, TICI $2 b-67 / 3$ in $97.4 \%$ (37/38), and TICI 2b/3 in 100\% (38/38) (50). The high FPE rate also translated into neurological improvement and better functional outcome with $92.1 \%$ of the cohort achieving NIHSS improvement of at least 4 points at $24 \mathrm{~h}$ and $81.6 \%$ having good outcomes (mRS 0-2) at discharge. There were no symptomatic intracerebral hemorrhages and at 90 days, the all-cause mortality was low at $5.3 \%$.

\section{Third-Generation Stent Retrievers}

The second-generation Solitaire and Trevo stent retrievers have been widely used and contributed greatly to the fact that EVT is now the standard of care for LVO AIS. However, this technology for stent retrievers has also evolved over time with a recent explosive improvement in the technology. It should be mentioned that despite this, randomized controlled trials showing an improvement in recanalization, functional outcomes, and reduced complications compared with existing stent retrievers have yet to be performed.

The EmboTrap reperfusion device (Neuravi/Cerenovus) is a third-generation stent retriever with a dual-layer structure furnished with articulating petals and a distal capture zone, supposed to enable better grip on the clot and entrapment of clot fragments generated by the EVT procedure (Figure 4).

The efficacy of the Embotrap stent was validated by an openlabel, single-arm, multicenter, prospective clinical trial conducted by the company which produced the stent, entitled Analysis of Revascularization in Ischemic Stroke with EmboTrap (ARISE II) which enrolled 227 patients (16). The mTICI $\geq 2 \mathrm{~b}$ reperfusion within three passes was achieved in $80.2 \%$, while the final mTICI $\geq 2$ b reperfusion rate was $92.5 \%$. Good functional outcome of

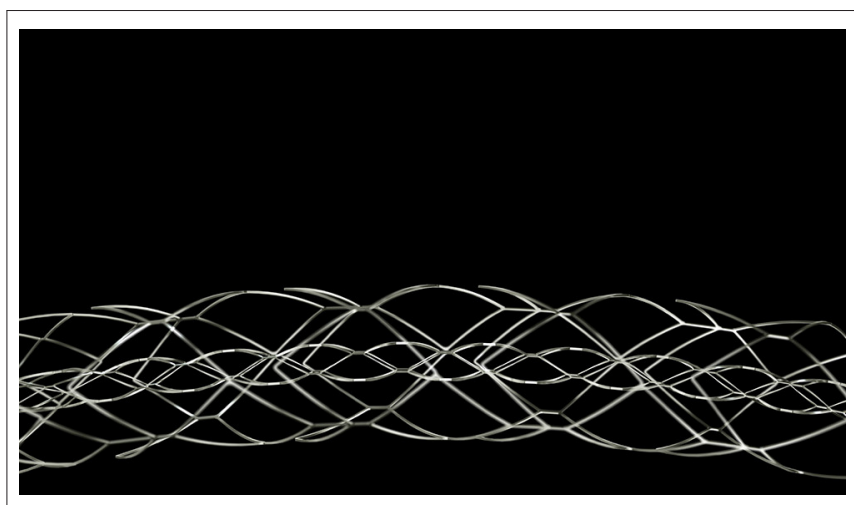

FIGURE 4 | EmboTrap reperfusion device (third-generation stent retriever) from Cerenovus (Miami, FL, USA).

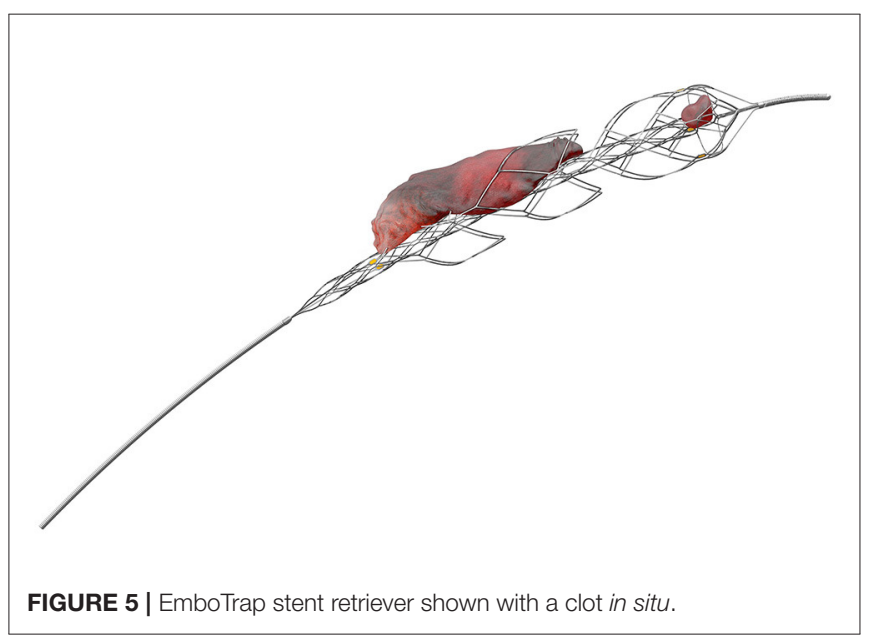

mRS $0-2$ at 90 days was achieved by $67 \%$ of the cohort, with a mortality rate of $9 \%$. Following this study, Embotrap was granted FDA approval for use in stroke thrombectomy. There are currently newer versions of the device, namely, the Embotrap 2 and Embotrap 3 in the market (Figure 5).

Another third-generation stent retriever is the threedimensional (3D) revascularization device (Penumbra Alameda, CA, USA). A multicentric randomized control trial with 198 patients was performed to evaluate the safety and efficacy of this device in combination with an intermediate catheter (51). Out of the 198 recruited patients, 98 underwent thrombectomy with the $3 \mathrm{D}$ stent retriever (Figure 6) in conjunction with an intermediate catheter and was able to achieve mTICI 2b-3 reperfusion in $81.9 \%$ of the patients. This rate was significantly higher than the comparison arm, where direct aspiration alone with an intermediate catheter achieved only $69.8 \%$ mTICI 2 b-3 reperfusion in 100 patients.

A new-generation stent retriever is the Versi, a nitinol stent retriever with two to four articulating segments expanding and reconfiguring under traction, during withdrawal, thus presumably facilitating clot trapping. An investigator-led clinical trial compared the third-generation Embotrap and Versi stents 


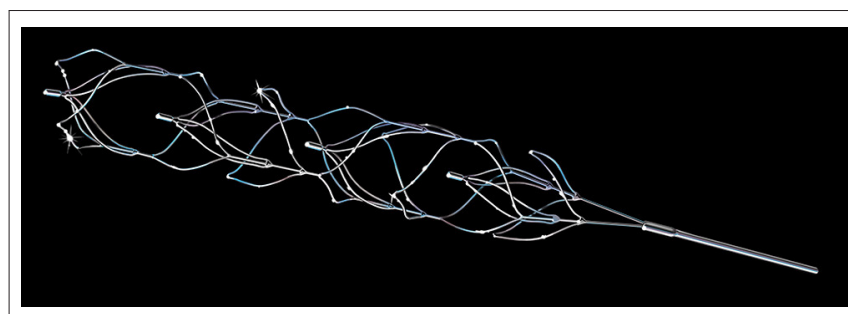

FIGURE 6 | Three-dimensional revascularization device from Penumbra.

with the earlier generation Solitaire and Trevo stent retrievers (52). They employed different flow models with various degrees of tortuosity to evaluate the different stent retrievers. The authors reported that the Versi had significantly better recanalization rates than the second-generation stent retrievers, while the Embotrap also had higher recanalization rates although not reaching statistical significance. The authors were able to discern that more severe tortuosity limited the effectiveness of earlier generation stent retrievers but not effectiveness of thirdgeneration stent retrievers. The finding could explain increased efficacy of third-generation stent retrievers. Nonetheless, this was a study performed in several flow models and remains to be validated in animal models as well as in clinical trials.

Another multisegmental stent retriever is known as the $\mathrm{NeVa}$ thrombectomy device. The $\mathrm{NeVa}$ device was designed to have an elevated radial force and has Drop Zones that help in the extraction of thrombi that adhere firmly to the artery wall. It is compatible with 0.021 in. microcatheters and is available in multiple sizes: M1-S $(4 \times 22 \mathrm{~mm}), \mathrm{M} 1(4 \times 30 \mathrm{~mm})$, and T $(4.5$ $\times 37 \mathrm{~mm}$ ) with the M1-S having no proximal "flow restoration zone" (53). The final segment is fashioned into a closed-ended basket, which retains the thrombus that has fallen into the Drop Zone openings. In an early single-center clinical trial with 118 patients, the rate of successful recanalization was $95.8 \%$ with firstpass mTICI $2 \mathrm{~b} / 3$ rates achieved in $56.8 \%$ of the patients and mTICI $2 \mathrm{c} / 3$ rates in $44.9 \%$ of the patients. Favorable functional outcome (modified Rankin Scale $0-2$ ) was seen in $42.4 \%$ of the population. The authors reported a $3.3 \% \mathrm{SICH}$ rate with the rate of embolization into new territory at $1.7 \%$ (53).

Another novel clot retriever is the Tigertriever (Rapid Medical, Yokneam, Israel) equipped with a handle-controlled mechanism allowing the operator to incrementally adjust the diameter of a nitinol-braided stent as well as collapse it. The feature of diameter adjustment is hypothesized to result in better wall apposition, robust clot integration, and variable exertion of radial force in different vascular segments $(54,55)$. Three versions of the Tigertriever device are available: The standard version (Tigertriever) has a net length of $32 \mathrm{~mm}$ (unexpanded form) and can expand up to $6 \mathrm{~mm}$ diameter, can be delivered through a microcatheter with an internal diameter of 0.021 in. In addition, a smaller version (Tigertriever 17) has a net length of $23 \mathrm{~mm}$ (unexpanded form) and can be delivered through a microcatheter with an internal diameter of $0.017 \mathrm{in}$. It can expand up to $3 \mathrm{~mm}$ diameter. Finally, there is a new Tigertriever 13 which can fit through a 0.013 -in. microcatheter. The Tigertriever device is CE approved since 2016 and completed several phase 1 trials (54-57). The investigators of the multicenter TIGER trial recently published their preliminary result. The Treatment With Intent to Generate Endovascular Reperfusion (TIGER) trial is a single-arm, prospective, multicenter trial comparing the Tigertriever to outcome in six recent pivotal studies (TREVO 2, SWIFT, MR CLEAN, ESCAPE, REVASCAT, and SWIFT PRIME) evaluating the Solitaire and Trevo stent-retriever devices. In the 160 enrolled patients treated with the Tigertriever, the primary efficacy end-point of mTICI $2 \mathrm{~b}-3$ reperfusion within three passes without use of rescue therapy was achieved in $84.6 \%$ in the main-study phase group compared with the $73.4 \%$ historical rate (non-inferiority $p<0.0001$; superiority $p<0.01$ ). Successful reperfusion (mTICI $\geq 2 b$ ) was achieved in $95.7 \%$ of the cohort and excellent reperfusion (mTICI $2 \mathrm{c}-3$ ) in $71.8 \%$. The primary safety composite end point rate of mortality and symptomatic intracranial hemorrhage was $18.1 \%$ compared with the $20.4 \%$ historical rate (non-inferiority $p=0.004$; superiority $p=0.57$ ). In the secondary outcome analysis, functional independence (mRS score $0-2$ ) was attained by $58.0 \%$, a rate superior to the $43.5 \%$ in the pooled comparator trials $(p=0.006)$ (58). The Tigertriever also appears promising in the treatment of distal, medium vessel occlusions (DMVOs) or as rescue treatment. In a cohort of 115 patients with both primary and secondary (after failed or incomplete EVT) DMVOs, as well as distal vessel thromboembolic complications during cerebral aneurysm or AVM embolization, mechanical thrombectomy performed with Tigertriever achieved successful recanalization in 86 patients (74.7\%) (59).

\section{ADJUNCTIVE TECHNIQUES}

\section{Rescue Stenting}

While the advent of the latest stent retrievers and large bore aspiration catheters have an increase of $90 \%$ reperfusion rates in LVO thrombectomy (Figure 7), there still exists a subset of patients whereby the EVT procedure fails to recanalize the vessel. Although there are several possible factors, one of the key reasons for failure of EVT is underlying intracranial atherosclerotic stenosis (ICAS). ICAS is commonly diagnosed during the EVT procedure by repeated recanalization and then acute re-occlusion of the vessel. In a Korean series of failed EVT, this occurred in up to $77 \%$ of the patient cohort (60). There are currently no guidelines on the optimum treatment in patients who failed thrombectomy and one possible technique is to perform permanent stenting to try to salvage the situation, in an attempt to keep the vessel patent. This is termed "rescue stenting" and was shown to be effective in an initial study of 45 failed thrombectomy patients, where the 17 patients with rescue stenting had better outcomes and less thirorcococn cerebral herniation than the 28 non-stenting patient (61).

Rescue stenting was validated in a large retrospective analysis of patients from 16 Korean stroke centers (62). In this study, patients with anterior circulation LVO who failed to recanalize following EVT were split into rescue stenting and nonrescue stenting groups. Out of 148 failed EVT patients, 48 received 


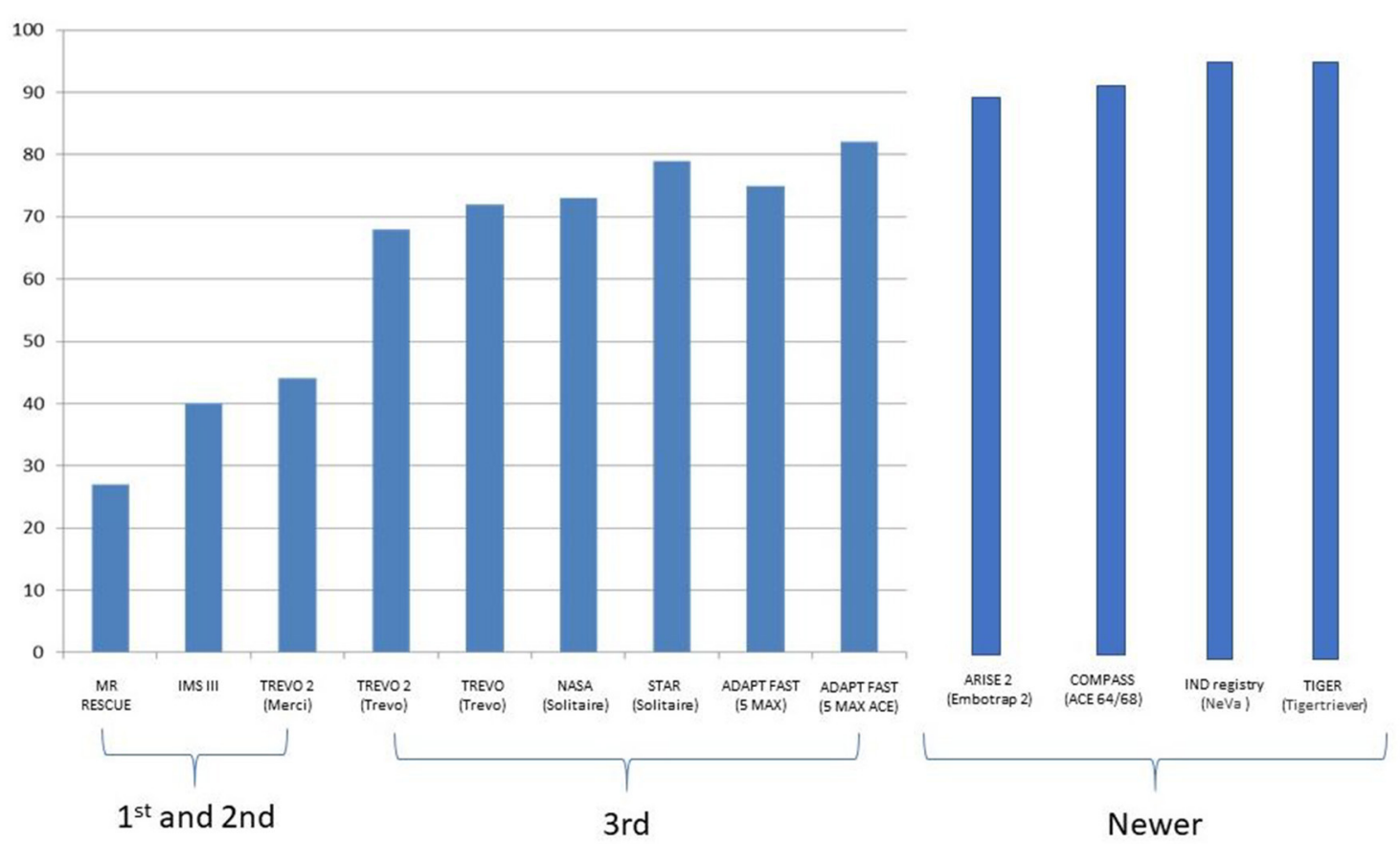

FIGURE 7 | Increasing MTICl 2b-3 reperfusions rates being reported with each successive generation of devices.

rescue stenting while 100 did not; $31 / 48$ rescue stenting patients (64.6\%) had successful mTICI $2 \mathrm{~b}-3$ reperfusion with rescue stenting, while none of the 100 patients without rescue stenting achieved reperfusion. Good functional outcome at 3 months was observed in $39.6 \%$ of the rescue stenting group and in $22.0 \%$ of the non-rescue stenting group ( $p=0.031$ ) without an increase in $\mathrm{SICH}$ or mortality. Of note, in the rescue stenting group who had successful reperfusion, $54.8 \%$ achieved good outcome despite the initial EVT failure, equivalent to the functional outcomes with mTICI 2b-3 reperfusion in the initial EVT attempts of $55.4 \%$. A meta-analysis on rescue stenting which included articles from 2015 to 2019, similarly found that in a sample of 352 patients, there was improved outcomes in the stenting arm compared with the refractory occlusion arm (OR, 2.87; 95\% CI, 1.77-4.66; $\left.p<0.001 ; I^{2}, 0 \%\right)$ with reduced mortality although there was some heterogeneity between studies for mortality (OR, 0.39; 95\% CI, $\left.0.16-0.93 ; p=0.03 ; I^{2}, 43 \%\right)$. The rates of SICH were not significantly different between both arms (63).

In a recent multinational study on emergency rescue stenting in AIS involving seven neurovascular centers (64), good outcome was observed in 73 of $163(44.8 \%)$ patients with recorded outcomes at 90 days. This is considerably better than the rates of $7 \%$ to $22 \%$ in cohorts with re-occlusion or persistent occlusion reports without rescue stenting. However, the rate of SICH in this analysis (11\%) was higher than in the aggregated thrombectomy studies without intracranial stenting of $4.4 \%$, and this was more common in anterior circulation occlusions than posterior circulation occlusions. A lower number of thrombectomy attempts before rescue stenting was also associated with better functional outcomes.

The choice to perform rescue stenting is difficult, as the permanent placement of a stent requires either acute glycoprotein $2 \mathrm{~b} / 3 \mathrm{a}$ inhibitors or dual antiplatelet treatment to prevent acute re-occlusion from in-stent thrombosis. In acute stroke patients with already sizable amounts of ischemic or infarcted tissue, these medications contribute to a potentially higher risk of symptomatic intracranial hemorrhage. Currently, there is little data and lack of consensus regarding antiplatelet management for intracranial stenting during thrombetomy. There is considerable variation in opinions even among the experts in this field. In an attempt to establish consensus on perioperative and postoperative antiplatelets management using the DELPHI method, a panel of 19 experts were surveyed. While the panel agreed that antiplatelet management in this setting should be standardized regardless of the size of the ischemic "core" on initial brain imaging or final perfusion result or treatment with intravenous alteplase, and that intravenous followed by oral aspirin is a possible choice, it failed to achieve consensus on several other important questions such as timing of initiation of therapy and the need for second antiplatelet agent and the choice of the second antiplatelet agent (65). Therefore, more data are needed to investigate the timing of initiation and choice of antiplatelet management in patients who undergo stenting in the setting of endovascular thrombectomy. Interestingly though, observational studies which have evaluated rescue stenting have demonstrated that the use of glycoprotein $2 \mathrm{~b} / 3 \mathrm{a}$ inhibitors did 
not significantly increase rates of SICH maintaining stent patency $(61,62,66-70)$.

\section{Fibrin Capsule of a Clot Broken by the Stent Retriever Followed by $2 b / 3 a$ Inhibitors}

For distal clots or clots past a tortuous intracranial segment, one technique is to deploy a stent retriever over the clot followed by administration of thrombolytics or glycoprotein $2 \mathrm{~b} / 3 \mathrm{a}$ inhibitor to dissolve the clot. The stent retriever is then resheathed in the microcatheter and removed, as there is a risk of avulsion of pial perforators with withdrawal of a deployed stent retriever. A small publication of 18 cases that initially failed thrombectomy, had a Solitaire stent retriever used as such with concomitant IA tirofiban, a glycoprotein $2 \mathrm{~b} / 3 \mathrm{a}$ inhibitor, administered. It showed successful reperfusion in 14 out of 18 patients $(77.7 \%)$ with good functional outcomes seen in $50 \%$ of the cases (69).

A more recent publication used a scanning electron microscope to review the structure of 199 thrombi extracted during thrombectomy procedures (71). Despite the heterogeneity of clot composition and organization, thrombi demonstrated a similar outer shell made of compacted fibrin, Von Willebrand factor, and platelets. This prevented the tPA from reaching the inner core of the thrombus, and hence preventing lysis. This study provides a potential explanation on how deploying a stent retriever is able to break the outer shell of the thrombus and expose the inner core to the $2 \mathrm{~b} / 3 \mathrm{a}$ inhibitor.

\section{IMMINENT QUESTIONS TO ANSWER}

\section{Large Ischemic Core}

While the current guidelines are clear that EVT should be performed for patients without a large early infarct or with an ASPECTS of six or more, there is a substantial proportion of patients who present to the hospital with an AIS with a sizable ischemic core. While the evidence is not clear, these patients may still derive some benefit from EVT. Pending the outcomes of randomized controlled trials, core-lab adjudicated pooled analyses of existing studies may shed preliminary insight into this crucial question. In one such study pooling data from seven randomized trials with a total of 1,764 patients, of which 871 were in the EVT arm and 893 in the best medical treatment arm, it was demonstrated that EVT was associated with better functional outcomes across a wide range of pretreatment imaging types on ordinal shift analyses (72). This included all ASPECTS groups except for ASPECTS 0-2 where the low sample size could not demonstrate statistical significance. However, this should be interpreted with caution, as in patients with a large ischemic burden or ASPECTS 4 or less, EVT was associated with significantly more SICH. Similarly, in the secondary analysis of the Optimizing Patient's Selection for Endovascular Treatment in Acute Ischemic Stroke (SELECT) trial, EVT was associated with better functional independence (mRS 0-2) compared with medical management alone (OR, 3.27; 95\% CI, 1.11-9.62; $p$ $=0.03)$. EVT was also associated with less infarct growth, and smaller final infarct volume than medical treatment (73).
Such analyses provide a strong foundation to support further investigation of the use of EVT for patients with large infarcts and poor ASPECTS at baseline. These initial results led to the ongoing SELECT-2 trial which is designed to evaluate thrombectomy compared with medical management in distal ICA and MCA M1 occlusions with a large core on either CT (ASPECTS 3-5) or advanced perfusion imaging $(\mathrm{rCBF}<30 \%$ or $\mathrm{ADC}<620$ or $50 \mathrm{ml}$ or more) and is expected to be completed at the end of 2021 (74). Other ongoing clinical trials, such as TENSION (NCT03094715), TESLA (NCT03805308), and IN EXTREMIS $(75,76)$ are also recruiting patients and will provide conclusive evidence on the use of EVT in patients with a large ischemic core at presentation.

\section{Clinically Mild Strokes With LVO}

A different group of patients are those who present with LVOs but mild strokes clinically. These are typically defined at NIHSS threshold of $<5$, where the risk of the EVT procedure needs to be weighed carefully against the potential benefit. A pooled data analysis of six comprehensive stroke centers with 300 patients having LVO and NIHSS 0-5, with 80 patients undergoing EVT and 220 patients undergoing best medical therapy was published (77). Of note is that the best medical treatment group allowed for rescue EVT if there was subsequent neurological deterioration. While the groups were not similar, EVT was associated with better functional outcomes (OR, 3.1; 95\% CI, 1.4-6.9) and in a propensity matched analysis, the superiority of EVT over best medical treatment persisted $(84.4 \%$ vs. $70.1 \% ; P=0.03)$.

Recently, another meta-analysis pooled patient data from 16 centers from 2013 to 2017 . This study evaluated 251 patients with LVO and mild stroke of which 138 were treated with EVT and 113 with best medical treatment. The study revealed that the 3-month functional outcomes were better in the best medical treatment group compared with the EVT group $(77.4 \%$ vs. $88.5 \% ; p=0.02)$ (78). The rate of asymptomatic ICH was also lower in the best medical management group as compared with the EVT group ( $4.6 \%$ vs. $17.5 \% ; p=0.002)$. The two groups did not differ in the rate of reperfusion or in safety outcomes.

We look forward to upcoming RCTs to further elucidate if mild strokes with LVO should be treated via EVT. One of these RCTs is ENDOLOW, which investigates anterior circulation occlusions with NIHSS scores $0-5$ and is enrolling patients in Canada, the USA, Germany, and Sweden (79). The INEXTREMIS trial also includes a substudy, which evaluates ischemic stroke patients with NIHSS $<6$ and LVO occlusions and similarly will be aiming to answer this important question (76).

\section{Very Late Presenting Patients: Acute Stroke Beyond $24 \mathrm{~h}$}

Evidence has recently emerged from the AURORA study, which pooled data from six randomized trials to examine effect of EVT in anterior circulation proximal LVO stroke from 6 to $24 \mathrm{~h}$ from time last seen well, that there is benefit of EVT in achieving reduced disability on functional outcome in terms of mRS in this group of patients, with an adjusted common odds ratio of 2.54 (95\% CI, 1.82-3.54; $p<0.0001$ ). In addition, the Number Needed to Treat to reduce mRS by 1 point was three patients. 
Furthermore, no significant differences in mortality or SICH were seen between EVT and control groups (80). This further substantiates the results of previous trials which elucidated that carefully selected patients benefit from thrombectomy up to $24 \mathrm{~h}$ $(81,82)$. However, there remains a pool of patients who present after $24 \mathrm{~h}$. At present, there are no clinical trials or guidelines that detail how we can manage these patients. Kim et al. examined the benefit of EVT in patients presenting very late. In their subgroup analysis of 150 patients who presented more than $16 \mathrm{~h}$ from their last known well time, EVT was performed only in 24 patients but a propensity matched analysis showed it was associated with increased odds of having favorable functional recovery at 3 months (adjusted OR, 11.08 (95\% CI, 1.88-108.60). In a further subgroup of patients $24 \mathrm{~h}$ from last known well, EVT was associated with favorable outcomes as well (adjusted OR, 10.54; 95\% CI, 2.18-59.34) (83). These preliminary studies substantiate the understanding that in patients with good collaterals, there is a chance to maintain the penumbra beyond $24 \mathrm{~h}$.

\section{EVT in Posterior Circulation Strokes}

There is much uncertainty related to treating posterior circulation stroke caused by basilar artery occlusion (BAO) with mechanical thrombectomy. The Basilar Artery International Cooperation study (BASICS) and endovascular treatment vs. standard medical treatment for vertebrobasilar artery occlusion (BEST) clinical trials are the only randomized clinical trials to date designed specifically to study the outcome of EVT in patients with basilar stroke within $6-8 \mathrm{~h}$ of onset $(84,85)$. However, the benefits of thrombectomy in the anterior circulation have not been replicated in basilar occlusions. These trials enrolled 300 patients and 131 patients respectively with more patients receiving IV tPA in the BASICS trial (80 vs. $30 \%$ ). Favorable functional outcome was defined as mRS 0 to 3 at 90 days, and this occurred in $44.2 \%$ in the endovascular group and $37.7 \%$ in the medical care group (risk ratio, $1.18 ; 95 \% \mathrm{CI}, 0.92$ to 1.50 ) in the BASICS trial, and $42 \%$ in the endovascular group vs. $32 \%$ in the medical group (adjusted OR, 1.74; 95\% CI, 0.813.74 ) in the BEST trial. In addition, SICH occurred in $4.5 \%$ of the patients after endovascular therapy and in $0.7 \%$ of those after medical therapy (risk ratio, $6.9 ; 95 \% \mathrm{CI}, 0.9$ to 53.0 ) in the BASICS trial and $8 \%$ in the endovascular arm and $0 \%$ in the control arm $(p=0.06)$ in the BEST trial. Nonetheless, it is worth noting that the investigators concluded that results of these trials may not exclude a substantial benefit of endovascular therapy as reflected by the wide confidence interval for the primary outcome. This is especially so considering that AIS secondary to $\mathrm{BAO}$ are often devastating for patients due to its high morbidity and mortality rates.

There are no randomized trials on thrombectomy in more distal posterior occlusions, and most of the pivotal trials excluded such patients. Strambo et al. examined the outcome of EVT in patient with isolated PCA occlusion vs. best medical therapy (BMT). They reported that complete recanalization at $24 \mathrm{~h}$ was achieved in $68 \%$ of patients undergoing EVT vs. $34.5 \%$ in BMT group ( $\mathrm{OR}=4.11 ; 95 \% \mathrm{CI}=1.35-12.53)$. This translated into a $15 \%$ absolute difference in the proportion of good outcome at 3 months in favor of the EVT group (55 vs. $40.5 \%$ ), and a $25 \%$ absolute difference in visual field normalization at 3 months (50 vs. $25.4 \%$ ) as well as a significantly better cognitive outcome with EVT (50 vs. 16.1\%). In terms of complications, the frequency of SICH and 3-month mortality was similar in both groups (86). The Thrombectomy for Primary Distal Posterior Cerebral Artery Occlusion Stroke or TOPMOST study was a multicentric case-control propensity-matched studies for primary occlusion of the PCA treated with EVT. In 184 matched patients, the NIHSS decreased by a mean of 2.4 points at discharge in the medical group and 3.9 points in the thrombectomy group (mean difference, -1.5 points; $95 \% \mathrm{CI}, 3.2$ to -0.8 ; $p=0.06$ ); this was balanced by an incidence of $4.3 \% \mathrm{SICH}$ in both arms (87).

\section{Direct to Thrombectomy Table - No IV tPA}

Time is an important variable that affects functional outcome in acute stroke thrombectomy and any delay in treatment initiation negatively impacts patients' functional outcomes (6).

While IV tPA has the ability to recanalize acute stroke occlusions, this has been eclipsed by the much superior recanalization rate of EVT. There is now a school of thought that instead of administering IV tPA, whether at an intervening primary stroke center or the comprehensive stroke center, patients with acute ischemic stroke from an LVO should go directly to endovascular thrombectomy. An RCT carried out in Japan entitled the SKIP trial comprised 200 AIS patients with anterior circulation occlusions presenting within $4 \mathrm{~h}$ of onset (88). At 3 months, the rate of good functional outcome was similar between the direct thrombectomy (59\%) and combined bridging approach (57\%). Furthermore, the mortality rate was similar between both arms as well. However, it was unable to prove noninferiority of direct to thrombectomy over bridging IV tPA $(0.6 \mathrm{mg} / \mathrm{kg}$ Japanese standardized dose $)$ because it was underpowered with a modest sample size. While the rate of asymptomatic hemorrhage was not significantly different between both arms and the rate of SICH was similarly nonsignificantly different, the combined rate of any ICH was significantly lower for the EVT group.

There were two other bridging IV tPA in thrombectomy trials which were similar in design to the SKIP trial but conducted across multiple stroke centers in China. The DIRECT-MT trial, in which 656 patients were enrolled, revealed that endovascular thrombectomy alone was non-inferior to combined intravenous alteplase and endovascular thrombectomy with regard to the functional outcome at 90 days (adjusted common odds ratio, 1.07 ; $95 \%$ confidence interval, 0.81 to $1.40 ; p=0.04$ for noninferiority). Of note, the non-inferiority margin was set at a high value of $20 \%$ margin of confidence in this trial (89). In the DEVT trial, the non-inferiority test also demonstrated that the endovascular thrombectomy alone was non-inferior to the combined IV thrombolysis and endovascular thrombectomy group $(z=2.7157, p$ for non-inferiority $=0.003)$ (90). This trial was terminated after first interim analysis in May 2020 as outcome measured crossed the pre-specified efficacy boundary. In this study, there was no significantly different rate of symptomatic ICH between groups; however, the rate 
of any ICH is significantly higher in the bridging r-TPA and thrombectomy group.

It is also worth noting that these trials were performed in east Asian populations, and further evidence is needed in a more diverse population, the SWIFT-DIRECT, MR CLEAN NOIV (91), and DIRECT-SAFE trials are upcoming international RCTs that can provide more information on the adoption of thrombectomy alone approach against bridging IV tPA (92).

\section{Stroke Secondary to Distal Medium Vessel Occlusion}

Endovascular thrombectomy is an evidence-based, guidelinerecommended treatment for acute ischemic stroke secondary to large vessel occlusion in the anterior circulation. However, endovascular treatment of DMVO is still unproven in view of the higher risk-benefit ratio with less severe clinical deficits and increased risk of iatrogenic complications. The exact definition of medium-sized vessel also requires consensus, as vessel size and anatomy may be subjected to interobserver variability, although a definition of medium vessel occlusion has been proposed using both anatomical characteristics and functional deficit (93).

Medium vessel occlusion was defined as occlusions of the M2/M3 middle cerebral artery/A2/A3 anterior cerebral artery and $\mathrm{P} 2 / \mathrm{P} 3$ posterior cerebral artery segments in a study with pooled data from two multicenter prospective cohorts. In this study, only $50.0 \%$ of patients with DMVO achieved an excellent outcome (mRS score, $0-1$ ) at 90 days and $67.4 \%$ achieved an independent outcome (mRS score, $0-2$ ). The authors did find that intravenous alteplase was significantly associated with lower mRS scores in mRS shift analysis, but there was no significant association with excellent outcome (mRS score, $0-1$ ). Moreover, even in the alteplase group, early recanalization was achieved in $<50 \%$ of study cohort, suggesting insufficient efficacy of intravenous alteplase as a stand-alone treatment for DMVO strokes (94).

There is some preliminary evidence from a meta-analysis of data from 12 nonrandomized studies which suggested that endovascular thrombectomy for patients with occlusions of M2 segment of Middle Cerebral Artery that can be safely accessed is associated with high recanalization rates and good clinical outcomes (95). In addition, a more recent meta-analysis of data from the HERMES Collaboration showed that for patients with M2 occlusions, treatment effect favored EVT over control (adjusted OR, 2.39; 95\% CI, 1.08 to 5.28 ; $p=0.03$ ) for $\mathrm{mRS}$ $0-2$ at 90 days, with number needed to treat for one patient to have functional independence (mRS 0-2) being 5.4 (96). There are also several single-center studies which provided encouraging evidence on the effect of endovascular thrombectomy in treating DMVO $(97,98)$, and there is hope that reperfusion rates with EVT could improve further with the use of smaller diameter next-generation stent retrievers and aspiration devices. We also wish to emphasize that direct aspiration thrombectomy in distal vessels such as the M3 or M4 has a risk of avulsion injury to the perforators and further studies are needed to determine their safety and effectiveness.

\section{Tandem Occlusions}

A tandem occlusion (TO), i.e., a thromboembolic obstruction in the intracranial cerebral vasculature in combination with an extracranial carotid artery occlusion, can occur in up to one-sixth of ischemic stroke patients (99). They tend not to have good recanalization rates with IV tPA and endovascular treatment is therefore advocated (100). In fact, subgroup analyses of the ESCAPE and MR CLEAN studies have suggested that patients with TO have better outcomes with early or concurrent treatment of the extracranial occlusion rather than later in a staged procedure $(101,102)$. Despite this, the optimal endovascular procedure in acute TO generally remains unclear. The controversy now is the optimal method of treating TO in acute stroke, i.e., is it better to initially bypass the extracranial occlusion and remove the intracranial occlusion first before returning to tackle the extracranial stenosis (the "retrograde" approach)? or is it preferable to attempt primary recanalization of the extracranial occlusion first, before moving on to treat the intracranial occlusion (the "antegrade" approach)?

The usual antegrade approach uses primary stenting to jail the extracranial stenotic atheromatous plaque, which should prevent showering of distal emboli (103). A theoretical drawback of the antegrade approach is the procedural time used for carotid stent placement which delays the time to intracranial reperfusion, which might result in an increase of the final infarct volume $(104,105)$. Furthermore, stent retriever-based thrombectomy techniques have the potential of entanglement between the struts of the stent retriever and carotid stent during withdrawal if the guiding catheter could not be advanced through the carotid stent. The retrograde approach achieves intracranial recanalization faster and some purport that this gives better functional outcomes (106); however, it may be difficult to pass through the proximal occlusion, and subsequent emboli from the proximal occlusion can sometimes re-occlude the intracranial circulation.

\section{Type of Anesthesia for Thrombectomy}

Anesthesia support is necessary for patients undergoing EVT particularly in complex anatomy, difficult cases, or in restless aphasic patients. The modality of anesthesia may have significant implications in outcomes for mechanical thrombectomy and each has its own proposed benefits. General anesthesia (GA) may improve procedural safety by keeping the patient still and protecting the airway, while conscious sedation (CS) has the benefit of neurologic monitoring with hemodynamic stability, and a quicker puncture time (107). It has been difficult to determine which is better for thrombectomy as the literature is very heterogeneous. The initial retrospective studies often based their anaesthesia choice on patient characteristics and what the operator was comfortable with. A retrospective study of 1,174 patients from 2009 to 2013 concluded that GA was inferior to CS; however, there was limited data on the important factors such as blood pressure and the NIHSS; moreover, their outcomes studied were only mortality and length of stay (108). Conversely, another retrospective database study of 2,512 patients concluded the opposite: that CS was superior to GA for stroke interventions. However, it too had many important factors lacking (109). 
In the more recent randomized trials attempting to address this topic, the GOLIATH (110), ANSTROKE (111), and SIETSA (112) trials reported GA and CS to be equally safe. In these trials, there was a prespecified target of systolic blood pressure of $>140 \mathrm{mmHg}$ prior to revascularization. A later analysis of these three studies showed that a blood pressure of $70 \mathrm{mmHg}$ or less was associated with a significantly worse functional outcomes. GA in those same studies had a higher incidence of mean arterial pressure decreases of $20 \%$ or more (113). The worse outcomes of GA with EVT may be explained by decreases in blood pressure and there may be a need to maintain the blood pressure when choosing the type of anesthesia. A more updated meta-analysis which included studies up to 2020 seems to support the RCT findings. This meta-analysis looked at 1,711 subjects undergoing GA and 1,961 subjects using CS. They found no significant difference between the two modalities for functional outcomes, recanalization, mortality, or complications although there was a trend toward SICH for GA (114).

Finally, local anesthesia alone without sedation is emerging as a possible alternative to conscious sedation. In a meta-analysis of 7,797 patients, there was no difference in functional outcomes between GA and LA or between CS and LA; however, there was a trend toward excellent functional outcome $(\mathrm{mRS} \leq 1)$ in the LA group vs. the GA group ( $\mathrm{OR}=1.44 ; 95 \% \mathrm{CI}, 1.00$ to $2.08 ; p=$ $0.05 ; I=70 \%)$ and a trend toward improved mortality in the LA group vs. the GA group (adjusted $\mathrm{OR}=1.24 ; 95 \% \mathrm{CI}, 1.00$ to 1.54 ;

\section{REFERENCES}

1. National Institute of Neurological Disorders and Stroke rt-PA Stroke Study Group. Tissue plasminogen activator for acute ischemic stroke. N Engl J Med. (1995) 333:1581-7. doi: 10.1056/NEJM199512143332401

2. Hacke W, Kaste M, Bluhmki E, Brozman M, Dávalos A, Guidetti D, et al. Thrombolysis with alteplase 3 to 45 hours after acute ischemic stroke. $N$ Engl J Med. (2008) 359:1317-29. doi: 10.1056/NEJMoa0804656

3. Goyal M, Menon BK, van Zwam WH, Dippel DWJ, Mitchell PJ, Demchuk AM, et al. Endovascular thrombectomy after large-vessel ischaemic stroke: a meta-analysis of individual patient data from five randomised trials Lancet. (2016) 387:1723-31. doi: 10.1016/S0140-6736(16)00163-X

4. Saver JL. Time is brain quantified. Stroke. (2006) 37:2636. doi: 10.1161/01.STR.0000196957.55928.ab

5. Saver JL, Fonarow GC, Smith EE, Reeves MJ, Grau-Sepulveda MV, Pan W, et al. Time to treatment with intravenous tissue plasminogen activator and outcome from acute ischemic stroke. JAMA. (2013) 309:24808. doi: 10.1001/jama.2013.6959

6. Saver JL, Goyal M, van der Lugt A, Menon BK, Majoie C, Dippel DW, et al. Time to treatment with endovascular thrombectomy and outcomes from ischemic stroke: a meta-analysis. JAMA. (2016) 316:127988. doi: 10.1001/jama.2016.13647

7. Powers WJ, Rabinstein AA, Ackerson T, Adeoye OM, Bambakidis $\mathrm{NC}$, Becker $\mathrm{K}$, et al. Guidelines for the early management of patients with acute ischemic stroke: 2019 update to the 2018 guidelines for the early management of acute ischemic stroke: a guideline for healthcare professionals from the American Heart Association/American Stroke Association. Stroke. (2019) 50:e344-418. doi: 10.1161/STR.0000000000000211

8. Bhogal P, Andersson T, Maus V, Mpotsaris A, Yeo L. Mechanical thrombectomy-a brief review of a revolutionary new treatment for thromboembolic stroke and the remaining questions. Clin Neuroradiol. (2018) 28: 313-26. doi: 10.1007/s00062-018-0692-2 $p=0.05 ; I=0 \%)$. The authors conclude that further anesthesia trials should have LA analyzed as a separate arm (115).

\section{CONCLUSION}

The field of thrombectomy in acute stroke continues to evolve rapidly, and periodic reviews of the literature are important. We present several of the evidence-based improvements to the procedure and pertinent issues that require further data to settle the controversy.

\section{AUTHOR CONTRIBUTIONS}

MJ was involved in data gathering, data analysis, drafting of the article, and is agreeable to be accountable for all aspects of the work. BT, PB, AG, CY, TT, FA, C-HS, SH, and TA was involved in the drafting of the article and the final approval. LY was involved in the conception and design of the project, data gathering, data analysis, drafting of the article, the final approval, and is agreeable to be accountable for all aspects of the work. All authors contributed to the article and approved the submitted version.

\section{FUNDING}

LY has received substantial grant funding from the National Medical Research Council (NMRC), Singapore and substantial support from the ministry of health $(\mathrm{MOH})$, Singapore.

9. Hong KS, Ko SB, Lee JS, Yu KH, Rha JH. Endovascular recanalization therapy in acute ischemic stroke: updated meta-analysis of randomized controlled trials. J Stroke. (2015) 17:268-81. doi: 10.5853/jos.2015.17.3.268

10. Song D, Cho AH. Previous and recent evidence of endovascular therapy in acute ischemic stroke. Neurointervention. (2015) 10:519. doi: 10.5469/neuroint.2015.10.2.51

11. Yoo AJ, Simonsen CZ, Prabhakaran S, Chaudhry ZA, Issa MA, Fugate JE, et al. Refining angiographic biomarkers of revascularization: improving outcome prediction after intra-arterial therapy. Stroke. (2013) 44:250912. doi: 10.1161/STROKEAHA.113.001990

12. Liebeskind D, Bracard S, Guillemin F, Jahan R, Jovin TG, Majoie C BLM, et al. eTICI reperfusion: defining success in endovascular stroke therapy. $J$ Neurointerv Surg. (2019) 11:433-8. doi: 10.1136/neurintsurg-2018-014127

13. Zaidat OO, Yoo AJ, Khatri P, Tomsick TA, von Kummer R, Saver JL, et al. Cerebral Angiographic Revascularization Grading (CARG) Collaborators, STIR Revascularization Working Group and STIR Thrombolysis In Cerebral Infarction (TICI) Task Force. Recommendations on angiographic revascularization grading standards for acute ischemic stroke: a consensus statement. Stroke. (2013) 44:2650-63. doi: 10.1161/STROKEAHA.113.001972

14. Rizvi A, Seyedsaadat SM, Murad MH, Brinjikji W, Fitzgerald ST, Kadirvel R, et al. Redefining 'success': a systematic review and meta-analysis comparing outcomes between incomplete and complete revascularization. J Neurointerv Surg. (2019) 11:9-13. doi: 10.1136/neurintsurg-2018-013950

15. García-Tornel Á, Requena M, Rubiera M, Muchada M, Pagola J, Rodriguez-Luna D, et al. When to stop. Stroke. (2019) 50:17818. doi: 10.1161/STROKEAHA.119.025088

16. Zaidat OO, Bozorgchami H, Ribó M, Saver JL, Mattle HP, Chapot $\mathrm{R}$, et al. Primary results of the multicenter ARISE II study (analysis of revascularization in ischemic stroke with EmboTrap). Stroke. (2018) 49:1107-15. doi: 10.1161/STROKEAHA.117.020125

17. Lapergue B, Blanc R, Gory B, Labreuche J, Duhamel A, Marnat G, et al. Effect of endovascular contact aspiration vs stent retriever on 
revascularization in patients with acute ischemic stroke and large vessel occlusion: the ASTER randomized clinical trial. JAMA. (2017) 318:44352. doi: 10.1001/jama.2017.9644

18. Turk AS III, Siddiqui A, Fifi JT, De Leacy RA, Fiorella DJ, Gu E, et al. Aspiration thrombectomy versus stent retriever thrombectomy as first-line approach for large vessel occlusion (COMPASS): a multicentre, randomised, open label, blinded outcome, non-inferiority trial. Lancet. (2019) 393:998-1008. doi: 10.1016/S0140-6736(19)30297-1

19. Ospel JM, Volny O, Jayaraman M, McTaggart R, Goyal M. Optimizing fast first pass complete reperfusion in acute ischemic stroke-the BADDASS approach (BAlloon guiDe with large bore distal access catheter with dual aspiration with Stent-retriever as Standard approach). Expert Rev Med Dev. (2019) 16:955-63. doi: 10.1080/17434440.2019.1684263

20. Goto S, Ohshima T, Ishikawa K, Yamamoto T, Shimato S, Nishizawa T, et al. A stent-retrieving into an aspiration catheter with proximal balloon (ASAP) technique: a technique of mechanical thrombectomy. World Neurosurg. (2018) 109:e468-75. doi: 10.1016/j.wneu.2017.10.004

21. Humphries W, Hoit D, Doss VT, Elijovich L, Frei D, Loy D, et al. Distal aspiration with retrievable stent assisted thrombectomy for the treatment of acute ischemic stroke. J Neurointerv Surg. (2015) 7:904. doi: 10.1136/neurintsurg-2013-010986

22. Maegerlein C, Mönch S, Boeckh-Behrens T, Lehm M, Hedderich DM, Berndt MT, et al. PROTECT: PRoximal balloon Occlusion TogEther with direCt Thrombus aspiration during stent retriever thrombectomy-evaluation of a double embolic protection approach in endovascular stroke treatment. J Neurointerv Surg. (2018) 10:751-5. doi: 10.1136/neurintsurg-2017-013558

23. Massari F, Henninger N, Lozano JD, Patel A, Kuhn AL, Howk M, et al. ARTS (Aspiration-Retriever Technique for Stroke): initial clinical experience. Interv Neuroradiol. (2016) 22:325-32 doi: 10.1177/1591019916632369

24. McTaggart RA, Tung EL, Yaghi S, Cutting SM, Hemendinger M, Gale HI, et al. Continuous aspiration prior to intracranial vascular embolectomy (CAPTIVE): a technique which improves outcomes. J Neurointerv Surg. (2017) 9:1154-9. doi: 10.1136/neurintsurg-2016-012838

25. Furlan A, Higashida R, Wechsler L, Gent M, Rowley H, Kase C, et al. Intraarterial prourokinase for acute ischemic stroke: the PROACT II study: a randomized controlled trial: prolyse in Acute Cerebral Thromboembolism. JAMA. (1999) 282:2003-11. doi: 10.1001/jama.282.21.2003

26. Khatri R, McKinney AM, Swenson B, Janardhan V. Bloodbrain barrier, reperfusion injury, and hemorrhagic transformation in acute ischemic stroke. Neurology. (2012) 79(Suppl. 1): S52-7. doi: 10.1212/WNL.0b013e3182697e70

27. Kaesmacher J, Bellwald S, Dobrocky T, Meinel TR, Piechowiak EI, Goeldlin M, et al. Safety and efficacy of intra-arterial urokinase after failed, unsuccessful, or incomplete mechanical thrombectomy in anterior circulation large-vessel occlusion stroke. JAMA Neurol. (2020) 77:31826. doi: 10.1001/jamaneurol.2019.4192

28. Zaidi SF, Castonguay AC, Jumaa MA, Malisch TW, Linfante I, Marden FA, et al. Intraarterial thrombolysis as rescue therapy for large vessel occlusions. Stroke. (2019) 50:1003-6. doi: 10.1161/STROKEAHA.118.024442

29. Yi TY, Chen WH, Wu YM, Zhang MF, Lin DL, Lin XH. Adjuvant intraarterial rt-PA injection at the initially deployed solitaire stent enhances the efficacy of mechanical thrombectomy in acute ischemic stroke. J Neurol Sci. (2018) 386:69-73. doi: 10.1016/j.jns.2018.01.012

30. Heiferman DM, Li DD, Pecoraro NC, Smolenski AM, Tsimpas A, Ashley WW Jr. Intra-arterial alteplase thrombolysis during mechanical thrombectomy for acute ischemic stroke. J StrokeCerebrovasc Dis. (2017) 26:3004-8. doi: 10.1016/j.jstrokecerebrovasdis.2017.07.031

31. Anadani M, Ajinkya S, Alawieh A, Vargas J, Chatterjee A, Turk A, et al. Intra-arterial tissue plasminogen activator is a safe rescue therapy with mechanical thrombectomy. World Neurosurg. (2019) 123:e6048. doi: 10.1016/j.wneu.2018.11.232

32. Clinicaltrials.gov. Multi-Arm Optimization of Stroke Thrombolysis (MOST). NCT03735979. Available online at: https://clinicaltrials.gov/ct2/show/ NCT03735979 (accessed April 15, 2021).

33. Chueh JY, Kühn AL, Puri AS, Wilson SD, Wakhloo AK, Gounis MJ, et al. Reduction in distal emboli with proximal flow control during mechanical thrombectomy: a quantitative in vitro study. Stroke. (2013) 44:1396401. doi: 10.1161/STROKEAHA.111.670463

34. Nikoubashman O, Wischer D, Hennemann HM, Sandmann J, Sichtermann T, Müschenich FS, et al. Balloon-guide catheters are needed for effective flow reversal during mechanical thrombectomy. AJNR Am J Neuroradiol. (2018) 39:2077-81. doi: 10.3174/ajnr.A5829

35. Nguyen TN, Castonguay AC, Nogueira RG, Haussen DC, English JD, Satti SR, et al. Effect of balloon guide catheter on clinical outcomes and reperfusion in Trevo thrombectomy. J NeuroInterv Surg. (2019) 11:8615. doi: 10.1136/neurintsurg-2018-014452

36. Nguyen TN, Malisch T, Castonguay AC, Gupta R, Sun CHJ, Martin CO, et al. Balloon guide catheter improves revascularization and clinical outcomes with the Solitaire device: analysis of the North American Solitaire acute stroke registry. Stroke. (2014) 45:141-5. doi: 10.1161/STROKEAHA.114.004828

37. Zaidat OO, Mueller-Kronast NH, Hassan AE, Haussen DC, Jadhav AP, Froehler MT, et al. Impact of balloon guide catheter use on clinical and angiographic outcomes in the STRATIS stroke thrombectomy registry. Stroke. (2019) 50:697-704. doi: 10.1161/STROKEAHA.118.022987

38. Brinjikji W, Starke RM, Murad MH, Fiorella D, Pereira VM, Goyal M, et al. Impact of balloon guide catheter on technical and clinical outcomes: a systematic review and meta-analysis. J Neurointerv Surg. (2018) 10:3359. doi: 10.1136/neurintsurg-2017-013179

39. Goyal M, Kappelhof M, Ospel JM, Bala F. Balloon guide catheters: use, reject, or randomize? Neuroradiology. (2021). 63:1179-83. doi: 10.1007/s00234-021-02739-2

40. Millikan JS, Cain TL, Hansbrough J. Rapid volume replacement for hypovolemic shock: a comparison of techniques and equipment. $J$ Trauma. (1984) 24:428-31. doi: 10.1097/00005373-198405000-00010

41. Hodge D, Fleisher G. Pediatric catheter flow rates. Am J Emerg Med. (1985) 3:403-7. doi: 10.1016/0735-6757(85)90198-6

42. Graber D, Dailey RH. Catheter flow rates updated. J Am Coll Emerg Phys. (1977) 6:518. doi: 10.1016/S0361-1124(77)80401-2

43. Bradley AG, Jadhav AP, Jovin TG, Jankowitz BT. Clinical comparison of new generation 0.071-inch and 0.072-inch aspiration catheters. World Neurosurg. (2019) 130:e463-6. doi: 10.1016/j.wneu.2019.06.120

44. Sanchez S, Bailey L, Ducore R, Andersson T, Nogueira R, Cognard C, et al. Preclinical evaluation of the ANCD thrombectomy device: safety and efficacy in a swine clot model. J Neurointerv Surg. (2020) 12:1008-13. doi: 10.1136/neurintsurg-2019-015548

45. Fitzgerald S, Ryan D, Thornton J, Nogueira RG. Preclinical evaluation of Millipede 088 intracranial aspiration catheter in cadaver and in vitro thrombectomy models. J NeuroInterv Surg. (2020) 13:447-52. doi: 10.1136/neurintsurg-2020-016218

46. Long TD, Kallmes DF, Hanel R, Shigematsu T, Halaszyn AM, Wolter J, et al. Novel aspiration catheter design for acute stroke thrombectomy. $J$ Neurointerv Surg. (2019) 11:190-5. doi: 10.1136/neurintsurg-2017-013702

47. Torabi R, Mokin M, Ren Z, Siddiqui A, Levy E, Waqas M, et al. E066 First U.S. experience with the $\mathrm{R}^{4} \mathrm{Q}$ distal access catheter for contact aspiration mechanical thrombectomy in emergent large vessel occlusion acute ischemic stroke. J NeuroInterv Surg. (2020) 12(Suppl. 1):A667. doi: 10.1136/neurintsurg-2020-SNIS.102

48. Simon S, Grey CP, Massenzo T, Simpson DG, Longest PW. Exploring the efficacy of cyclic vs static aspiration in a cerebral thrombectomy model: an initial proof of concept study. J Neurointerv Surg. (2014) 6:67783. doi: 10.1136/neurintsurg-2013-010941

49. Arslanian RA, Marosfoi M, Caroff J, King RM, Raskett C, Puri AS, et al. Complete clot ingestion with cyclical ADAPT increases first-pass recanalization and reduces distal embolization. J NeuroInterv Surg. (2019) 11:931-6. doi: 10.1136/neurintsurg-2018-014625

50. Kalousek V, Yoo A, Sheth S, Janardhan V, Mamic J, Janardhan V. Cyclical aspiration using a novel mechanical thrombectomy device is associated with a high TICI 3 first pass effect in large-vessel strokes. J Neuroimaging. (2021). doi: 10.1111/jon.12889. [Epub ahead of print].

51. Nogueira RG, Frei D, Kirmani JF, Zaidat O, Lopes D, Turk AS III, et al. Safety and efficacy of a 3-dimensional stent retriever with aspiration-based thrombectomy vs aspiration-based thrombectomy alone in acute ischemic stroke intervention: a randomized clinical trial. JAMA Neurol. (2018) 75:304-11. doi: 10.1001/jamaneurol.2017.3967

52. Kaneko N, Komuro Y, Yokota H, Tateshima S. Stent retrievers with segmented design improve the efficacy of thrombectomy in tortuous vessels. J NeuroInterv Surg. (2019) 11:119-22. doi: 10.1136/neurintsurg-2018-014061

53. Akpinar CK, Ozdemir AO, Gurkas E, Bilgic AB, Aykac O, Inanc Y, et al. Favorable first-pass recanalization rates with $\mathrm{NeVa}^{\mathrm{TM}}$ thrombectomy device 
in acute stroke patients: initial clinical experience. Interv Neuroradiol. (2020) 2:1591019920938223. doi: 10.1177/1591019920938223

54. Will L, Maus V, Maurer C, Weber A, Weber W, Fischer S. Mechanical thrombectomy in acute ischemic stroke using a manually expandable stent retriever (Tigertriever): preliminary single center experience. Clin Neuroradiol. (2020) 31:491-7. doi: 10.1007/s00062-020-00919-w

55. Kara B, Selcuk HH, Salik AE, Zalov H, Yildiz O, Gul G, et al. Singlecenter experience with the Tigertriever device for the recanalization of large vessel occlusions in acute ischemic stroke. J Neurointerv Surg. (2019) 11:455-9. doi: 10.1136/neurintsurg-2018-014196

56. Kara B, Selcuk HH, Yildiz O, Cetinkaya D. Revascularization of acute basilar artery occlusion using the tigertriever adjustable clot retriever. Clin Neuroradiol. (2017) 27:241-3. doi: 10.1007/s00062-016-0532-1

57. Gruber P, Diepers M, von Hessling A, Weber J, Kahles T, Anon J, et al. Mechanical thrombectomy using the new Tigertriever in acute ischemic stroke patients-a Swiss prospective multicenter study. Interv Neuroradiol. (2020) 27:1591019920946499. doi: 10.1177/1591019920946499

58. Gupta R, Saver J, Levy E, Zaidat OO, Yavagal D, Liebeskind DS, et al. New class of radially adjustable stentrievers for acute ischemic strokeprimary results of the multicenter TIGER trial. Stroke. (2021) 52:153444. doi: 10.1161/STR.0000000000000376

59. Rikhtegar R, Mosimann PJ, Weber R, Wallocha M, Yamac E, Mirza-Aghazadeh-Attari M, et al. Effectiveness of very low profile thrombectomy device in primary distal medium vessel occlusion, as rescue therapy after incomplete proximal recanalization or following iatrogenic thromboembolic events. J NeuroIntervent Surg. (2021). doi: 10.1136/neurintsurg-2020-017035. [Epub ahead of print].

60. Baek JH, Kim BM, Kim DJ, Heo JH, Nam HS, Song D, et al. Importance of truncal-type occlusion in stentriever-based thrombectomy for acute stroke. Neurology. (2016) 87:1542-50. doi: 10.1212/WNL.0000000000003202

61. Baek JH, Kim BM, Kim DJ, Heo JH, Nam HS, Yoo J. Stenting as a rescue treatment after failure of mechanical thrombectomy for anterior circulation large artery occlusion. Stroke. (2016) 47:23603. doi: 10.1161/STROKEAHA.116.014073

62. Chang Y, Kim BM, Bang OY, Baek J, Heo JH, Nam HS, et al. Rescue stenting for failed mechanical thrombectomy in acute ischemic stroke: a multicenter experience. Stroke. (2018) 49:958-64. doi: 10.1161/STROKEAHA.117.020072

63. Premat K, Dechartres A, Lenck S, Shotar E, Le Bouc R, Degos V, et al. Rescue stenting versus medical care alone in refractory large vessel occlusions: a systematic review and meta-analysis. Neuroradiology. (2020) 62:62937. doi: $10.1007 / \mathrm{s} 00234-020-02360-9$

64. Stracke CP, Fiehler J, Meyer L, Thomalla G, Krause LU, Lowens $\mathrm{S}$, et al. Emergency intracranial stenting in acute stroke: predictors for poor outcome and for complications. J Am Heart Assoc. (2020) 9:e012795. doi: 10.1161/JAHA.119.012795

65. Goyal M, Orlov K, Jensen ME, Taylor A, Majoie C, Jayaraman M, et al. A DELPHI consensus statement on antiplatelet management for intracranial stenting due to underlying atherosclerosis in the setting of mechanical thrombectomy. Neuroradiology. (2021) 63:627-32. doi: 10.1007/s00234-020-02556-z

66. Baek JH, Kim BM, Heo JH, Kim DJ, Nam HS, Yong DK. Endovascular and clinical outcomes of vertebrobasilar intracranial atherosclerosis-related large vessel occlusion. Front Neurol. (2019) 10:215. doi: 10.3389/fneur.2019.00215

67. Park H, Baek JH, Kim BM. Endovascular treatment of acute stroke due to intracranial atherosclerotic stenosis-related large vessel occlusion. Front Neurol. (2019) 10:308. doi: 10.3389/fneur.2019.00308

68. Kang DH, Kim YW, Hwang YH, Park SP, Kim YS, Baik SK, et al. Instant reocclusion following mechanical thrombectomy of in situ thromboocclusion and the role of low-dose intra-arterial tirofiban. Cerebrovasc Dis. (2014) 37:350-5. doi: 10.1159/000362435

69. Seo JH, Jeong HW, Kim ST, Kim EG. Adjuvant tirofiban injection through deployed solitaire stent as a rescue technique after failed mechanical thrombectomy in acute stroke. Neurointervention. (2015) 10:227. doi: 10.5469/neuroint.2015.10.1.22

70. Delgado F, Oteros R, Jimenez-Gomez E, Rey IB, Bautista MD, Moyano RV. Half bolus dose of intravenous abciximab is safe and effective in the setting of acute stroke endovascular treatment. J Neurointerv Surg. (2019) 11:147-52. doi: 10.1136/neurintsurg-2018-014163
71. Di Meglio L, Desilles JP, Ollivier V, Nomenjanahary MS, Meglio SD, Deschildre C, et al. Acute ischemic stroke thrombi have an outer shell that impairs fibrinolysis. Neurology. (2019) 93:e1686-98. doi: 10.1212/WNL.0000000000008395

72. Román LS, Menon BK, Blasco J, Hernández-Pérez M, Dávalos A, Majoie CBLM, et al. Imaging features and safety and efficacy of endovascular stroke treatment: a meta-analysis of individual patient-level data. Lancet Neurol. (2018) 17:895-904. doi: 10.1016/S1474-4422(18)30242-4

73. Saaraj A, Hassan A, Savitz S, Sitton C, Grotta J, Chen P, et al. Outcomes of endovascular thrombectomy vs medical management alone in patients with large ischemic cores: a secondary analysis of the optimizing patient's selection for endovascular treatment in acute ischemic stroke (SELECT) study. JAMA Neurol. (2019) 76:1147-56. doi: 10.1001/jamaneurol.2019.2109

74. Clinicaltrials.gov. A Randomized Controlled Trial to Optimize Patient's Selection for Endovascular Treatment in Acute Ischemic Stroke (SELECT2). NCT03876457. Available online at: https://clinicaltrials.gov/ct2/show/ NCT03876457 (accessed April 15, 2021).

75. Bendszus M, Bonekamp S, Berge E, Boutitie F, Brouwer P, Gizewski E, et al. A randomized controlled trial to test efficacy and safety of thrombectomy in stroke with extended lesion and extended time window. Int J Stroke. (2019) 14:87-93. doi: 10.1177/1747493018798558

76. MOSTE LASTE. In Extremis Study. Available online at: https://www. inextremis-study.com (accessed April 15, 2021).

77. Nagel S, Bouslama M, Krause LU, Küpper C, Messer M, Petersen M, et al. Mechanical thrombectomy in patients with milder strokes and large vessel occlusions. Stroke. (2018) 49:2391-7. doi: 10.1161/STROKEAHA.118.021106

78. Goyal N, Tsivgoulis G, Malhotra K, Ishfaq MF, Pandhi A, Frohler MT, et al. Medical management vs mechanical thrombectomy for mild strokes: an international multicenter study and systematic review and meta-analysis. JAMA Neurol. (2019) 77:16-24. doi: 10.1001/jamaneurol.2019.3112

79. Clinicaltrials.gov. Endovascular Therapy for Low NIHSS Ischemic Strokes (ENDOLOW) NCT04167527. Available online at: https://clinicaltrials.gov/ ct2/show/NCT04167527 (accessed April 15, 2021).

80. Jovin T, Lansberg M, Hill M, Goyal M, Mocco J, Turk A. Thrombectomy for anterior circulation stroke beyond 6 hours from time last known well: Final results of the Aurora (analysis of pooled data from randomized studies of thrombectomy more than 6 hours after last known well) collaboration. In: Presented at International Stroke Conference: Abstract LB 8. (2021).

81. Nogueira RG, Jadhav AP, Haussen DC, Bonafe A, Budzik R, Bhuva P, et al. Thrombectomy 6 to 24 hours after stroke with a mismatch between deficit and infarct. N Engl J Med. (2018) 378:11-21. doi: 10.1056/NEJMoa1706442

82. Albers GW, Marks MP, Kemp S, Christensen S, Tsai JP, Ortega-Gutierrez S, et al. Thrombectomy for stroke at 6 to 16 hours with selection by perfusion imaging. N Engl J Med. (2018) 378:708-18. doi: 10.1056/NEJMoa1713973

83. Kim BJ, Menon BK, Kim JY, Shin DW, Baik SH, Jung C, et al. Endovascular treatment after stroke due to large vessel occlusion for patients presenting very late from time last known well. JAMA Neural. (2021) 78:219. doi: 10.1001/jamaneurol.2020.2804

84. Langezaal L, van Der Hoeven E, MontÁlverne F, de Carvalho JJF, Lima FO, Dippel DWJ, et al. Endovascular therapy for stroke due to basilarartery occlusion. N Engl J Med. (2021) 384:1910-20. doi: 10.1056/NEJMoa20 30297

85. Liu X, Dai Q, Ye R, Zi W, Liu Y, Wang H, et al. Endovascular treatment versus standard medical treatment for vertebrobasilar artery occlusion (BEST): an open-label, randomised controlled trial. Lancet Neurol. (2020) 19:11522. doi: 10.1016/S1474-4422(19)30395-3

86. Strambo D, Bartolini B, Beaud V, Marto JP, Sirimarco G, Dunet V, et al. Thrombectomy and thrombolysis of isolated posterior cerebral artery occlusion cognitive, visual, and disability outcomes. Stroke. (2020) 51:25461. doi: 10.1161/STROKEAHA.119.026907

87. Meyer L, Stracke CP, Jungi N, Wallocha M, Broocks G, Sporns $\mathrm{PB}$, et al. Thrombectomy for primary distal posterior cerebral artery occlusion stroke: the TOPMOST study. JAMA Neurol. (2021) 78:43444. doi: 10.1001/jamaneurol.2021.0001

88. Suzuki K, Kimura K, Takeuchi M, Morimoto M, Kanazawa R, Kamiya $\mathrm{Y}$, et al. The randomized study of endovascular therapy with versus without intravenous tissue plasminogen activator in acute stroke with ICA and M1 occlusion (SKIP study). Int J Stroke. (2019) 14:7525. doi: $10.1177 / 1747493019840932$ 
89. Yang P, Zhang Y, Zhang L, Zhang Y, Treurniet KM, Chen W, et al. Endovascular thrombectomy with or without intravenous Alteplase in acute stroke. N Engl J Med. (2020) 382:1981-93. doi: 10.1056/NEJMoa2001123

90. Zi W, Qiu Z, Li F, Sang H, Wu D, Luo W, et al. Effect of endovascular treatment alone vs intravenous Alteplase plus endovascular treatment on functional independence in patients with acute ischemic stroke: The DEVT Randomized Clinical Trial. JAMA. (2021) 325:23443. doi: 10.1001 /jama.2020.23523

91. Treurniet KM, LeCouffe NE, Kappelhof M, Emmer BJ, van Es A, Boiten J, et al. MR CLEAN-NO IV: intravenous treatment followed by endovascular treatment versus direct endovascular treatment for acute ischemic stroke caused by a proximal intracranial occlusion-study protocol for a randomized clinical trial. Trials. (2021) 22:141. doi: 10.1186/s13063-021-05063-5

92. clinicaltrials.gov. Bridging Thrombolysis Versus Direct Mechanical Thrombectomy in Acute Ischemic Stroke (SWIFT DIRECT) NCT03192332. Available online at: https://clinicaltrials.gov/ct2/show/NCT03192332 (accessed April 15, 2021)

93. Goyal M, Ospel JM, Menon BK, Hill MD. MeVO: the next frontier? J Neurointerv Surg. (2020) 12:545-7. doi: 10.1136/neurintsurg-2020-015807

94. Ospel JM, Menon BK, Demchuk AM, Almekhlafi MA, Kashani N, Mayank A, et al. Clinical course of acute ischemic stroke due to medium vessel occlusion with and without intravenous alteplase treatment. Stroke. (2020) 51:3232-40. doi: 10.1161/STROKEAHA.120.030227

95. Saber H, Narayanan S, Palla M, Saver JL, Nogueira RG, Yoo AJ, et al. Mechanical thrombectomy for acute ischemic stroke with occlusion of the M2 segment of the middle cerebral artery: a meta-analysis. J Neurointerv Surg. (2018) 10:620-4. doi: 10.1136/neurintsurg-2017-013515

96. Menon BK, Hill MD, Davalos A, Roos Y, Campbell BCV, Dippel D WJ, et al. Efficacy of endovascular thrombectomy in patients with M2 segment middle cerebral artery occlusions: meta-analysis of data from the HERMES collaboration. J Neurointerv Surg. (2019) 11:10659. doi: 10.1136/heartjnl-2014-307109.258

97. Pérez-García C, Rosati S, Gómez-Escalonilla C, López-Frías A, Arrazola J, Moreu M. MeVO SAVE technique: initial experience with the $167 \mathrm{~cm}$ long NeuroSlider 17 for a combined approach in medium vessel occlusions (MeVOs). J Neurointerv Surg. (2020) 13:768. doi: 10.1136/neurintsurg-2020-016763

98. Pérez-García C, Moreu M, Rosati S, Simal P, Egido JA, Gomez-Escalonilla $\mathrm{C}$, et al. Mechanical thrombectomy in medium vessel occlusions: blind exchange with mini-pinning technique versus mini stent retriever alone. Stroke. (2020) 51:3224-31. doi: 10.1161/STROKEAHA.120.030815

99. Goyal M, Demchuk AM, Menon BK, Eesa M, Rempel J, Thorton J, et al. Randomized assessment of rapid endovascular treatment of ischemic stroke. N Engl J med. (2015) 372:1019-30. doi: 10.1056/NEJMoa1414905

100. Del Zoppo GJ, Poeck K, Pessin MS, Wolpert SM, Furlan AJ, Ferbert A, et al. Recombinant tissue plasminogen activator in acute thrombotic and embolic stroke. Ann Neurol. (1992) 32:78-86. doi: 10.1002/ana.410320113

101. Berkhemer OA, Borst J, Kappelhof M, Yoo AJ. van dan Berg LA, Fransen PSS, et al. Extracranial carotid disease and effect of intra-arterial treatment in patients with proximal anterior circulation stroke in MR CLEAN. Ann Int Med. (2017) 166:867-75. doi: 10.7326/M16-1536

102. Assis Z, Menon BK, Goyal M, Demchuk AM, Shankar J, Rempel JL, et al. Acute ischemic stroke with tandem lesions: technical endovascular management and clinical outcomes from the ESCAPE trial. J Neurointerv Surg. (2018) 10:429-33. doi: 10.1136/neurintsurg-2017-01 3316

103. Rubiera M, Ribo M, Delgado-Mederos R, Santamarina E, Delgado $\mathrm{P}$, Montaner J, et al. Tandem internal carotid artery/middle cerebral artery occlusion: an independent predictor of poor outcome after systemic thrombolysis. Stroke. (2006) 37:23015. doi: 10.1161/01.STR.0000237070.80133.1d

104. Lockau H, Liebig T, Henning $T$, Neuschmelting V, Stetefeld $H$, Kabbasch C, et al. Mechanical thrombectomy in tandem occlusion: procedural considerations and clinical results. Neuroradiology. (2015) 57:589-98. doi: 10.1007/s00234-014-1465-5

105. Marnat G, Mourand I, Eker O, Machi P, Arquizan C, Riquelme C, et al. Endovascular management of tandem occlusion stroke related to internal carotid artery dissection using a distal to proximal approach: insight from the RECOST study. AJNR Am J Neuroradiol. (2016) 37:12818. doi: 10.3174/ajnr.A4752

106. Yang D, Shi Z, Lin M, Zhou Z, Zi W, Wang H, et al. Endovascular retrograde approach may be a better option for acute tandem occlusions stroke. Interv Neuroradiol. (2019) 25:194-201. doi: 10.1177/1591019918805140

107. Eker OF, Saver J, Goyal M, Jahan R, Levy EI, Nogueira RG, et al. Impact of anesthetic management on safety and outcomes following mechanical thrombectomy for ischemic stroke in SWIFT PRIME cohort. Front Neurol. (2018) 9:702. doi: 10.3389/fneur.2018.00702

108. Bekelis K, Missios S, MacKenzie T, Tjoumakaris S, Jabbour P. Anesthesia technique and outcomes of mechanical thrombectomy in patients with acute ischemic stroke. Stroke. (2017) 48:3616. doi: 10.1161/STROKEAHA.116.015343

109. McDonald JS, Brinjikji W, Rabinstein A, Cloft HJ, Lanzino G, Kallmes DF. Conscious sedation versus general anaesthesia during mechanical thrombectomy for stroke: a propensity score analysis. J Neurointerv Surg. (2015) 7:789-94. doi: 10.1136/neurintsurg-2014-011373

110. Simonsen CZ, Yoo A, Sørensen L, Juul N, Johnsen S, Andersen G, et al. Effect of general anesthesia and conscious sedation during endovascular therapy on infarct growth and clinical outcomes in acute ischemic stroke: a randomized clinical trial. JAMA Neurol. 75:470-7. doi: 10.1001/jamaneurol.2017.4474

111. Löwhagen Hendén P, Rentzos A, Karlsson J, Rosengren L, Leiram $B$, Sundeman $H$, et al. General anesthesia versus conscious sedation for endovascular treatment of acute ischemic stroke: the an stroke trial (anesthesia during stroke). Stroke. (2017) 48:1601-7. doi: 10.1161/STROKEAHA.117.016554

112. Schönenberger S, Möhlenbruch M, Pfaff J, Mundiyanapurath S, Kieser M, Bendszus M, et al. Sedation vs. Intubation for endovascular stroke treatment (SIESTA) - a randomized monocentric trial. Int J Stroke. (2015) 10:96978. doi: $10.1111 /$ ijs. 12488

113. Zhang Y, Lu J, Fang F, Ma L, Cai B, Faramand A. General anesthesia versus conscious sedation for intracranial mechanical thrombectomy: a systematic review and meta-analysis of randomized clinical trials. J Am Heart Assoc. (2019) 8:e011754. doi: 10.1161/JAHA.118. 011754

114. Shen $\mathrm{H}, \mathrm{Ma} \mathrm{X}, \mathrm{Wu} \mathrm{Z}$, Shao X, Cui J, Zhang B, et al. Conscious sedation compared to general anesthesia for intracranial mechanical thrombectomy: a meta-analysis. Brain Behav. (2021) 11:e02161. doi: 10.1002/ brb3.2161

115. Butt W, Dhillon PS, Podlasek A, Malik L, Nair S, Hewson D, et al. Local anesthesia as a distinct comparator versus conscious sedation and general anesthesia in endovascular stroke treatment: a systematic review and metaanalysis. J Neurointerv Surg. (2021). doi: 10.1136/neurintsurg-2021-017360. [Epub ahead of print].

Conflict of Interest: TA is a consultant for Ablynx, Amnis Therapeutics, Medtronic, Cerenovus/J\&J, Rapid Medical and Anaconda. AG is a consultant for Stryker, Medtronic, Penumbra and has received educational grants from Abbott. $\mathrm{PB}$ is consultant for Phenox.

The remaining authors declare that the research was conducted in the absence of any commercial or financial relationships that could be construed as a potential conflict of interest.

Publisher's Note: All claims expressed in this article are solely those of the authors and do not necessarily represent those of their affiliated organizations, or those of the publisher, the editors and the reviewers. Any product that may be evaluated in this article, or claim that may be made by its manufacturer, is not guaranteed or endorsed by the publisher.

Copyright (c) 2021 Yeo, Jing, Bhogal, Tu, Gopinathan, Yang, Tan, Arnberg, Sia, Holmin and Andersson. This is an open-access article distributed under the terms of the Creative Commons Attribution License (CC BY). The use, distribution or reproduction in other forums is permitted, provided the original author(s) and the copyright owner(s) are credited and that the original publication in this journal is cited, in accordance with accepted academic practice. No use, distribution or reproduction is permitted which does not comply with these terms. 